\title{
Alois Urban
}

Théorème fondamental de la théorie du contact des courbes

Czechoslovak Mathematical Journal, Vol. 7 (1957), No. 2, 273-294

Persistent URL: http://dml.cz/dmlcz/100247

\section{Terms of use:}

(C) Institute of Mathematics AS CR, 1957

Institute of Mathematics of the Czech Academy of Sciences provides access to digitized documents strictly for personal use. Each copy of any part of this document must contain these Terms of use.

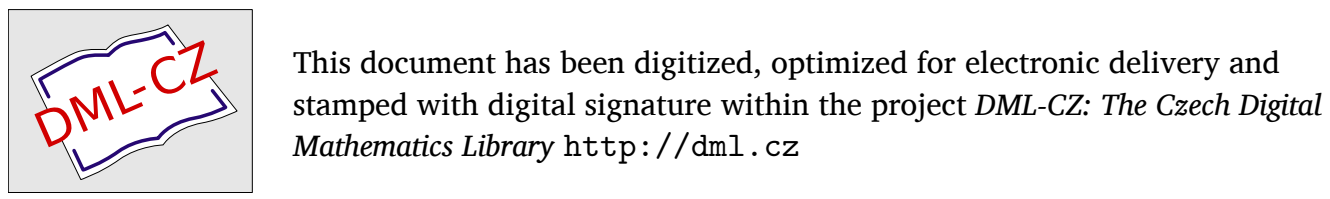




\title{
THÉORĖME FONDAMENTAL DE LA THÉORIE DU CONTACT DES COURBES
}

\author{
ALOIS URBAN, Praha. \\ (Reçu le 23 mai 1956.)
}

\begin{abstract}
En employant la notion du contact analytique des courbes au sens de M. Fubini, l'auteur traite une extension d'un théorème dû à M. $\breve{C E C H}_{\mathrm{E}}$ qui exprime des conditions nécessaires et suffisantes pour que deux courbes, ayant au point commun, qui est un point simple de chacune d'eux, un contact d'ordre $s-1(s \geq 1)$, aient en réalité au point donné un contact d'ordre $s+\sigma-1(\sigma \geqq 1)$. La condition restrictive $\sigma \leqq s$, posée par M. Cech, n'est pas supposée.
\end{abstract}

1. Introduction. En cherchant la solution générale de deux problèmes fondamentaux de la théorie du contact des courbes, c'est-à-dire l'étude du contact des projections de deux courbes d'un centre de projection ${ }^{1}$ ) et l'étude du contact des projections d'une courbe de deux centres différents, $\left.{ }^{2}\right)$ M. E. С̆ECH a utilisé avec avantage un théorème sur le contact des courbes, qu'il avait formulé et démontré. $\left.{ }^{3}\right)$ Cet énoncé donne des conditions nécessaires et suffisantes pour que les courbes supposées d'avoir au point commun, qui est un point simple de l'une et de l'autre courbe, un contact d'ordre $s-1(s \geqq 1)$, aient en réalité au point donné un contact d'ordre $s+\sigma-1(\sigma \geqq 1)$.

Le théorème fondamental ayant été énoncé pour le cas $1 \leqq \sigma \leqq s$ seulement, on devait donc poser de même cette condition restrictive en chercheant la solution générale de deux problèmes fondamentaux mentionnés plus haut.

En généralisant le théorème dû à M. E. Čech, nous énonçons dans ce Mémoire un théorème général où n'intervient pas la supposition restrictive $\sigma \leqq s$. En considérant l'importance de cet énoncé dans la solution de divers problèmes concernants le contact des courbes,' on peut le regarder comme théorème fondamental de la théorie du contact des courbes.

\footnotetext{
1) $[3]$.

2) $[1] ;[2]$, p. 153-161; [4].

$\left.{ }^{3}\right)$ [2]; p. 116--117; [3], p. 6-10.
} 
2. Théorème fondamental de la théorie du contact des courbes dans l'espace euclidien. Soient données, dans un espace linéaire $E_{n}$ à $n$ dimensions $(n \geqq 2)$ rapporté à un système de coordonnées curvilignes $u_{i}(i=1, \ldots, n)$, deux courbes $C_{1}, C_{2}$ definies respectivement par des équations paramétriques

$$
C_{1} \equiv u_{i}=\varphi_{i}(w), \quad C_{2} \equiv u_{i}=\psi_{i}(v), \quad(i=1, \ldots, n),
$$

où $\varphi_{i}(w)\left(\psi_{i}(v)\right)(i=1, \ldots, n)$ sont des fonctions univoques et analytiques d'une variable indépendante $w(v)$, définies dans un intervalle ouvert $W(V)$.

Considérons sur la courbe $C_{1}\left(C_{2}\right)$ un point simple arbitraire $A(B)$ correspondant à la valeur $v=\stackrel{\circ}{w} \epsilon W(v=\stackrel{v}{\epsilon} \epsilon V)$ du paramètre $w(v)$ et désignons

$$
\varphi_{i v}=\left[\frac{\mathrm{d}^{v} \varphi_{i}(w)}{\mathrm{d} w^{\nu}}\right]_{w=i v}, \quad \psi_{i v}=\left[\frac{\mathrm{d}^{v} \psi_{i}(v)}{\mathrm{d} v^{\nu}}\right]_{v=0}, \quad(i=1, \ldots, n ; v=0,1,2, \ldots),
$$

où, comme d'ordinaire, pour $v=0$ on pose $\varphi_{i 0}=p_{i}(\stackrel{\circ}{w}), \psi_{i 0}=\psi_{i}(\stackrel{\circ}{v}) ; A(B)$ étant un point simple de la courbe $C_{1}\left(C_{2}\right)$, tous les nombres $\varphi_{i 1}\left(\psi_{i 1}\right)$ ne peuvent. pas s'annuler simultanément.

Supposons maintenant que les points $A, B$ soient confondus, c'est-à-dire soit $\varphi_{i 0}=\psi_{i 0}(i=1, \ldots, n)$.

Cela étant, nous déduissons le théorème fondamental suivant:

Théorème 1. Soient $s \geqq 1, \sigma \geqq 1$ des nombres entiers. Supposons que les courbes $C_{1}, C_{2}$ aient au point $A$ un contact analytique d'ordre $s-1^{4}$ )

Les courbes $C_{1}, C_{2}$ ont au point $A$ un contact d'ordre $s+\sigma-1^{5}$ ) si et seulement si l'on peut trouver des nomb̈res $a_{\nu}\left(v=0,1, \ldots, \sigma-1 ; a_{0} \neq-1\right.$ dans le cas $s=1$ ) tels que

$$
\begin{gathered}
\psi_{i, s+t}-\varphi_{i, s+t}=\sum_{k=1}^{k^{\prime}} \sum_{t_{1}=0}^{t_{n}} \sum_{t_{2}=0}^{t_{1}} \ldots \sum_{t_{k}=0}^{t_{k-1}} \frac{\varphi_{i, t_{k}+k}}{k !} a_{t_{0}-t_{1}} a_{t_{1}-t_{2}} \ldots a_{t_{k-1}-t_{k}} \\
\cdot\left(\begin{array}{c}
k s+t_{0} \\
(k-1) s+t_{1}
\end{array}\right)\left(\begin{array}{c}
(k-1) s+t_{1} \\
(k-2) s+t_{2}
\end{array}\right) \ldots\left(\begin{array}{c}
2 s+t_{k-2} \\
s+t_{k-1}
\end{array}\right)\left(\begin{array}{c}
s+t_{k-1} \\
t_{k}
\end{array}\right), \\
(i=1, \ldots, n ; t=0, \ldots, \sigma-1),
\end{gathered}
$$

où $t_{0}$ est donné par la relation $t_{0}=t-(k-1) s$ et où $k_{0}, k^{\prime}$ sont des nombres entiers déterminés de manière que $\left(k_{0}-1\right) s+1 \leqq \sigma \leqq k_{0} s, 1 \leqq k^{\prime} \leqq k_{0}$, $\left.\left(k^{\prime}-1\right) s \leqq t<k^{\prime} s .{ }^{6}\right)$

Démonstration. Selon la supposition, les deux courbes $C_{1}$ et $C_{2}$ ont au point commun $A$ un contact analytique d'ordre $s-1(s \geqq 1)$. Il s'ensuit de la définition du contact analytique que par rapport à la correspondance donnée par la relation $w=v$ on a pour le paramètre $\stackrel{\circ}{w}=\stackrel{\circ}{v}$ du point $A$

$$
\varphi_{i v}=\psi_{i v} \quad(i=1, \ldots, n ; v=0, \ldots, s-1) .
$$

4) La définition du contact analytique d'ordre $s-1$ est donné p. ex. dans [4], p. 3-4.

$\left.{ }^{5}\right)$ On entend par là un contact géométrique.

${ }^{5}$ ) [4], p. 6-8 (pour le cas $k_{0}=1$ ). 
On voit sans difficulté que toutes les correspondances analytiques qui réalisent un contact d'ordre $s-1$, peuvent être mises sous la forme suivante

$$
w=v+\sum_{\alpha=0}^{\infty} \frac{(v-v)^{s+\alpha}}{(s+\alpha) !} a_{\alpha}
$$

étant donné $\left(\frac{\mathrm{d} w}{\mathrm{~d} v}\right)_{v=\dot{v}} \neq 0$, dans le cas $s=1$ nous supposons $a_{0} \neq-1$.

La condition nécessaire et suffisante pour que les courbes $C_{1}$ et $C_{2}$ aient un contact d'ordre $s+\sigma-1(\sigma \geqq 1)$ au point $A$ est qu'il existe une correspondance analytique

$$
w=F(v)
$$

telle que $F\left(\stackrel{\circ}{)}=\stackrel{\circ}{v}\left(\frac{\mathrm{d} F}{\mathrm{~d} v}\right)_{v=0} \neq 0\right.$ et telle que le contact analytique des courbes $C_{1}$ et $C_{2}$ au point $A$ par rapport à cette correspondance soit d'ordre $s+$ $+\sigma-1$, c'est-à-dire que

$\left[\frac{\mathrm{d}^{v} \varphi_{i}(F(v))}{\mathrm{d} v^{\nu}}\right]_{v=0}=\left[\frac{\mathrm{d}^{v} \psi_{i}(v)}{\mathrm{d} v^{v}}\right]_{v=0}, \quad(i=1, \ldots, n ; v=0, \ldots, s+\sigma-1)$.

Pour la correspondance (2.4) on en déduit, ayant égard aux équations (2.2) la relation

$$
\left(\frac{\mathrm{d} F}{\mathrm{~d} v}\right)_{v=i}=1
$$

en supposant $s \geqq 2$, et les relations ultérieures

$$
\left(\frac{\mathrm{d}^{v} F}{\mathrm{~d} v^{v}}\right)_{v=0}=0 \quad(v=2, \ldots, s-1)
$$

en supposant $s \geqq 3$.

Il est évident, d'aprês les conditions (2.6) et (2.7) justement trouvées que chacune de ces correspondances appartient aux correspondances (2.3); elle a donc la forme

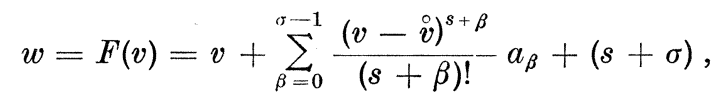

où $(m)$ désigne l'ensemble des membres qui ont $(v-\stackrel{\circ}{v})^{m}$ comme facteur.

Afin de pouvoir trouver les conditions nécessaires et suffisantes démandées telles que le contact des courbes $C_{1}$ et $C_{2}$ soit d'ordre $s+\sigma-1$ au point $A$, il faut substituer (2.8) à (2.5).

Avant tout, nous allons trouver $\varphi_{i}(F(v))$. On a

$$
\varphi_{i}(F(v))=\varphi_{i 0}+\sum_{\alpha=1}^{s+\sigma-1} \frac{\varphi_{i \alpha}}{\alpha !}(F(v)-\stackrel{\circ}{v})^{\alpha}+(s+\sigma),
$$


où il faut encore substituer pour $(F(v)-\stackrel{\circ}{v})^{\alpha}, \alpha=1, \ldots, s+\sigma-1$; pour $(F(v)-v)^{\alpha}$ on trouve

$$
\begin{aligned}
&(F(v)-\stackrel{o}{v})^{\alpha}=\left(v-\stackrel{o}{v}+\sum_{\beta=0}^{\sigma-1} \frac{(v-\stackrel{\circ}{v})^{s+\beta}}{(s+\beta) !} a_{\beta}\right)^{\alpha}+(s+\sigma)= \\
&= \sum_{k=v}^{\alpha}\left(\begin{array}{l}
\alpha \\
k
\end{array}\right)(v-\stackrel{o}{v})^{\alpha+k(s-1)}\left(\sum_{p=0}^{\sigma-1} \frac{(v-\stackrel{\circ}{v})^{\beta}}{(s+\beta) !} a_{\beta}\right)^{k}+(s+\sigma)= \\
&=(v-\stackrel{o}{v})^{\alpha}+\sum_{k=1}^{\alpha} \sum_{\beta_{1}, \ldots, \beta_{k}=0}^{\sigma-1}\left(\begin{array}{l}
\alpha \\
k
\end{array}\right) \frac{a_{\beta_{1}} a_{\beta_{2}} \ldots a_{\beta_{k}}}{\left(s+\beta_{1}\right) !\left(s+\beta_{2}\right) ! \ldots\left(s+\beta_{k}\right) !} . \\
&(v-\stackrel{o}{v})^{\alpha+k(s-1)+\beta_{1}+\beta_{2}+\ldots+\beta_{k}}+(s+\sigma), \\
&(\alpha=1, \ldots, s+\sigma-1) .
\end{aligned}
$$

On peut mettre le résultat obtenu sous une forme où $(s+\sigma)$ désignerait tous les membres de la fonction $(F(v)-\stackrel{\circ}{v})^{\alpha}$, qui possèdent au moins $(v-\stackrel{o}{v})^{s+\sigma}$ comme facteur.

Introduisons, pour abréger, une nouvelle notation

$$
\begin{gathered}
M_{\alpha, k}=\sum_{\beta_{1}, \beta_{2}, \ldots, \beta_{k}=0}^{\sigma-1}\left(\begin{array}{c}
\alpha \\
k
\end{array}\right) \frac{a_{\beta_{1}} a_{\beta_{2}} \ldots a_{\beta_{k}}}{\left(s+\beta_{1}\right) !\left(s+\beta_{2}\right) ! \ldots\left(s+\beta_{k}\right) !}(v-\stackrel{o}{v})^{\alpha+k(s-1)+\beta_{1}+\beta_{2}+\ldots+\beta_{k}} \\
(\alpha=1, \ldots, s+\sigma-1 ; k=1, \ldots, \alpha) .
\end{gathered}
$$

Les nombres entiers $s \geqq 1, \sigma \geqq 1$ étant donnés, nous pouvons trouver sans ambiguité un nombre entier positif de telle manière que les inégalités

soient satisfaites.

$$
\left(k_{0}-1\right) s+1 \leqq \sigma \leqq k_{0} s
$$

Enfin, désignons par $\sigma_{\lambda}$ les nombres entiers définis par la relation

$$
\sigma_{\lambda}=\sigma-(\lambda-1)(s-1),
$$

où $\lambda$ est un nombre entier (particulièrement $\sigma_{0}=s+\sigma-1, \sigma_{1}=\sigma$ ). On voit immédiatement que les nombres $\sigma_{\lambda}$ possèdent les propriétés suivantes
a) $s=1 \Longleftrightarrow \sigma_{\lambda}=\sigma$ pour chaque $\lambda$,
b) $s>1, \lambda<\mu \Longleftrightarrow \sigma_{\lambda}>\sigma_{\mu}$,
c) $\sigma_{k_{0}+1} \leqq k_{0} \leqq \sigma_{k_{0}}$.

En vertu de (2.11) la condition $\alpha+k(s-1)+\sum_{i=1}^{k} \beta_{i}>s+\sigma-1$ équivaut à l'inégalité

$$
\sum_{i=1}^{k} \beta_{i}>\sigma_{k}-\alpha
$$

or, il est évident, que $(s+\sigma)$ contient précisément tous les membres de $M_{\alpha, k}(\alpha=1, \ldots, s+\sigma-1 ; k=1, \ldots, \alpha)$ pour lesquels les conditions (2.14) sont vérifiées. La condition $\sum_{i=1}^{k} \beta_{i} \geqq 0$ étant toujours satisfaite, il en résulte que 
pour chaque nombre entier positif $k$ et pour chaque $\alpha>\sigma_{k}$ tous les membres de $M_{\alpha, k}$ appartiennent à $(s+\sigma)$; or particulièrement tous les membres de $M_{\alpha, k}$ pour $k>k_{0}$ appartiennent de même à $(s+\sigma)$, parce que, d'après (2.12), dans ce cas on a toujours $\alpha>\sigma_{k}$.

Maintenant, nous pouvons trouver aisément l'expression pour $\varphi_{i}(F(v))$; il suffit de substituer (2.10) dans l'équation (2.9) et d'employer les résultats justement trouvés.

On déduit

$$
\begin{aligned}
& \varphi_{i}(F(v))=\sum_{\beta=0}^{s+\sigma-1} \frac{\varphi_{i / \beta}}{\beta !}(v-\stackrel{o}{v})^{\beta}+\sum_{k=1}^{k_{n}} \sum_{\alpha=k}^{\sigma_{k}} \sum_{\substack{\beta_{1}, \beta_{2}, \ldots, \beta_{k}=0 \\
\left(\beta_{1}+\beta_{2}+\ldots+\beta_{k} \leqq \sigma_{k}-\alpha\right)}}^{\sigma-1} \frac{\bar{\varphi}_{i \alpha}}{\alpha !} . \\
& \cdot\left(\begin{array}{l}
\alpha \\
k
\end{array}\right) \frac{a_{\beta_{1}} \ldots a_{\beta_{k}}}{\left(s+\beta_{1}\right) ! \ldots\left(s+\beta_{k}\right) !}(v-\stackrel{0}{v})^{\alpha+k(s-1)+\beta_{1}+\ldots+\beta_{k}}+(s+\sigma) .
\end{aligned}
$$

Quant au résultat trouvé, on peut le remplacer par une autre équation plus convenable. A cet effet, nous ferons usage de l'identité

$$
\begin{gathered}
\frac{1}{\alpha !}\left(\begin{array}{l}
\alpha \\
k
\end{array}\right) \frac{1}{\left(s+\beta_{1}\right) ! \ldots\left(s+\beta_{k}\right) !}=\frac{1}{k !} \frac{1}{\left(\alpha+k(s-1)+\beta_{1}+\ldots+\beta_{k}\right) !} . \\
. \frac{\left((\alpha-k)+\left(s+\beta_{1}\right)+\ldots+\left(s+\beta_{k}\right)\right) !}{(\alpha-k) !\left(s+\beta_{1}\right) ! \ldots\left(s+\beta_{k}\right) !}= \\
=\frac{1}{k !} \frac{1}{\left(\alpha+k(s-1)+\beta_{1}+\ldots+\beta_{k}\right) !}\left(\begin{array}{c}
\alpha-k+s+\beta_{1} \\
s+\beta_{1}
\end{array}\right) . \\
.\left(\begin{array}{c}
\alpha-k+2 s+\beta_{1}+\beta_{2} \\
s+\beta_{2}
\end{array}\right) \ldots\left(\begin{array}{c}
\alpha+k(s-1)+\beta_{1}+\ldots+\beta_{k} \\
s+\beta_{k}
\end{array}\right) .
\end{gathered}
$$

En outre on peut encore remplacer les paramètres $\alpha, \beta_{i}(i=1, \ldots, k)$ par les nouveaux paramètres $t_{\lambda}(\lambda=0, \ldots, k)$ introduits moyennant les équations

$$
t_{k}=\alpha-k, \quad t_{i}=\alpha-k+\sum_{\lambda=1}^{k-i} \beta_{\lambda} \quad(i=0, \ldots, k-1)
$$

et par le paramètre auxiliaire $t$ introduit moyennant l'équation

$$
t=t_{0}+(k-1) s .
$$

Des conditions $k \leqq \alpha \leqq \sigma_{k}, 0 \leqq \beta_{i} \leqq \sigma-1 \quad(i=1, \ldots, k), \sum_{i=1}^{k} \beta_{i} \leqq \sigma_{k}-\alpha$ et de $(2.13 a, b)$ il résulte que

$$
0 \leqq \sum_{\lambda=1}^{i} \beta_{\lambda} \leqq \sigma_{k}-\alpha \quad(i=1, \ldots, k)
$$

Or, pour les paramètres $\hat{i}_{\lambda}(\lambda=0,1, \ldots, k)$ et $t$, on obtient $0 \leqq t_{0} \leqq \sigma_{k}-k, \quad 0 \leqq t_{i+1} \leqq t_{i} \quad(i=0, \ldots, k-1),(k-1) s \leqq t \leqq \sigma-1$. 
En substituant (2.16), (2.17) et (2.18) dans (2.15) on obtient en définitive

$$
\begin{aligned}
& \varphi_{i}(F(v))=\sum_{\alpha=0}^{s-1} \frac{\varphi_{i x}}{\alpha !}(v-v)^{\alpha}+\sum_{t=0}^{\sigma-1} \frac{\varphi_{i, s+t}}{(s+t) !}(v-v)^{s+t}+\sum_{k=1}^{k_{1}} \sum_{t=(k-1) s}^{\sigma-1} \sum_{t_{1}}^{t_{n}} \sum_{t_{2}=0}^{t_{1}} \ldots \\
& \ldots \sum_{t_{k}=0}^{t_{k-1}} \frac{\varphi_{i, t_{k}+k}}{k !} \frac{(v-v)^{s+t}}{(s+t) !} \prod_{j=0}^{k-1} a_{t_{k-j-1}-t_{k-j}}\left(\begin{array}{c}
(k-j) s+t_{j} \\
\left.(k-j-1) s+t_{j+1}\right)
\end{array}\right)+(s+\sigma) .
\end{aligned}
$$

De (2.19) il est facile de déterminer $\left(\frac{\mathrm{d}^{s+t} \varphi_{i}(F(v))}{\mathrm{d} v^{s+t}}\right)_{v=0}$ pour $0 \leqq t \leqq \sigma-1$. Si nous choisissons un nombre entier positif $k^{\prime}$ tel que $1 \leqq k^{\prime} \leqq k_{0}$, alors. nous trouvons

$$
\begin{aligned}
\left(\frac{\mathrm{d}^{s+t} \varphi_{i}(F(v))}{\mathrm{d} v^{s+t}}\right)_{v-j}=\varphi_{i, s+t}+\sum_{k=1}^{k^{\prime}} \sum_{t_{1}=0}^{t_{0}} \sum_{t_{2}=0}^{t_{1}} \ldots \sum_{t_{k}=0}^{t_{k-1}} \frac{\varphi_{i, t_{k}+k}}{k !} a_{t_{0}-t_{1}} a_{t_{1}-t_{2}} \ldots a_{t_{k-1}-t_{k}} . \\
\cdot\left(\begin{array}{c}
k s+t_{0} \\
(k-1) s+t_{1}
\end{array}\right)\left(\begin{array}{c}
(k-1) s+t_{1} \\
(k-2) s+t_{2}
\end{array}\right) \ldots\left(\begin{array}{c}
s+t_{k-1} \\
t_{k}
\end{array}\right)
\end{aligned}
$$

pour $t$ qui satisfait aux inégalités $\left(k^{\prime}-1\right) s \leqq t<k^{\prime} s$.

En substituant (2.20) dans (2.5) on obtient (2.1), c.q.f.d.

Remarque. D'après (2.13a), dans le cas où $s=1$, nous avons $k_{0}=\sigma, k^{\prime}=$ $=t+1, t_{0}=t+1-k$ et par suite nous pouvons mettre (2.1) sous la forme $\left(a_{0} \neq-1\right)$

$$
\begin{gathered}
\psi_{i, 1+t}-\phi_{i, 1+t}=\sum_{k=1}^{t+1} \sum_{t_{1}=0}^{t+1-k} \sum_{t_{2}=0}^{t_{1}} \ldots \sum_{t_{k}=0}^{t_{k}} \frac{\varphi_{i, t k+k}}{k !} a_{t+1-k-t_{1}} a_{t_{1}-t_{2}} \ldots a_{t_{k-1}-t_{k}} . \\
\cdot\left(\begin{array}{c}
t+1 \\
k-1+t_{1}
\end{array}\right)\left(\begin{array}{l}
k-1+t_{1} \\
k-2+t_{2}
\end{array}\right) \ldots\left(\begin{array}{c}
2+t_{k-2} \\
1+t_{k-1}
\end{array}\right)\left(\begin{array}{c}
1+t_{k-1} \\
t_{k}
\end{array}\right) .
\end{gathered}
$$

3. Théorème fondamental de la théorie du contact des courbes dans un espace projectif. Soit donné un espace projectif $S_{n}$ à $n$ dimensions $(n \geqq 2)$ rapporté à un système de coordonnées homogènes ordinaires (linéaires) $x^{(i)}$ $(i=0,1, \ldots, n)$.

Dans l'espace $S_{n}$, soient données deux courbes $\left.{ }^{7}\right) C_{1}$ et $C_{2}$ définies respectivement par les équations paramétriques

$$
C_{1} \equiv x^{(i)}=x^{(i)}(w), \quad C_{2} \equiv y^{(i)}=y^{(i)}(v), \quad(i=0,1, \ldots, n),
$$

que nous allons écrire, comme d'habitude, en forme vectorielle

$$
\text { a) } C_{1} \equiv x=x(w), \quad \text { b) } C_{2} \equiv y=y(v) \text {. }
$$

$\left.{ }^{7}\right)$ Les équations $z^{(i)}=z^{(i)}(t),(i=0,1, \ldots, n)$, expriment les équations d'une courbe

a) si les fonctions $z^{(i)}(t), i=0,1, \ldots, n$, sont des fonctions d'une variable indépendante $t$ définies dans un intervalle ouvert $T$,

b) si un au moins des nombres $z^{(0)}, z^{(1)}, \ldots, z^{(n)}$ est différent de zéro pour chaque valeur du paramètre $t$ de l'intervalle $T$,

c) si, $a^{(i)}$ étant des constantes arbitraires, $\varrho(t) \neq 0$ étant une fonction univoque analytique du paramètre $t$, les équations $z^{(i)}=\varrho(t) a^{(i)}(i=0,1, \ldots, n)$ ne sont pas. vérifiées. 
Soit $A$ un point simple de la courbe $C_{1}\left(C_{2}\right)$ correspondant au paramètre $w=\stackrel{\circ}{w}(v=\stackrel{\circ}{v})$.

Pour les fonctions $x(w)$ et $y(v)$ posons

$$
x_{v}=\left[\frac{\mathrm{d}^{v} x(w)}{\mathrm{d} w^{v}}\right]_{w=\dot{w}}, \quad y_{v}=\left[\frac{\mathrm{d}^{v} y(v)}{\mathrm{d} v^{\nu}}\right]_{v=i}, \quad(v=0,1, \ldots),
$$

où $x_{0}=x(\stackrel{\circ}{w}), y_{0}=y(\stackrel{\circ}{v})$.

Nous allons énoncer le théorème suivant:

Théorème 2. Soient $s \geqq 1, \sigma \geqq 1$ des nombres entiers. Soit $k_{0}$ un nombre entier positif donné par les inégalités $\left(k_{0}-1\right) s+1 \leqq \sigma \leqq k_{0} s$.

Supposons que les courbes $C_{1}$ et $C_{2}$ aient au point $A$ un contact analytique d'ordre $\left.s-1 ;{ }^{8}\right)$ soit $y_{v}=x_{v}(v=0,1, \ldots, s-1)$.

Les courbes $C_{1}$ et $C_{2}$ ont au point $A$ un contact d'ordre $s+\sigma-1^{9}$ ) si et seulement si l'on peut trouver des nombres $a_{v}, b_{v}\left(v=0,1, \ldots, \sigma-1 ; a_{0} \neq-1\right.$ pour $s=1$ ) tels que

1. $k_{0}=1$ :

$y_{s+\alpha}-x_{s+\alpha}=\sum_{v=0}^{\alpha}\left(\begin{array}{c}s+\alpha \\ v\end{array}\right)\left(a_{\alpha-\nu} x_{\nu+1}+b_{\alpha-\nu} x_{\nu}\right), \quad(\alpha=0,1, \ldots, \sigma-1)$;

2. $k_{0}>1$ :

a) $y_{s+\alpha}-x_{s+\alpha}=\sum_{\nu=0}^{\alpha}\left(\begin{array}{c}s+\alpha \\ v\end{array}\right)\left(a_{\alpha-\nu} x_{v+1}+b_{\alpha-\nu} x_{\nu}\right), \quad(\alpha \doteq 0,1, \ldots, s-1)$,

b) $y_{2 s+\alpha}-x_{2 s+\alpha}=\sum_{\nu=0}^{s+\alpha}\left(\begin{array}{c}2 s+\alpha \\ v\end{array}\right)\left(a_{s+\alpha-\nu} x_{\nu+1}+b_{s+\alpha-\nu} x_{\nu}\right)+\sum_{k=2}^{k^{\prime}} \sum_{t_{1}=0}^{t_{0}} \sum_{t_{2}=0}^{t_{1}} \ldots$

$$
\begin{aligned}
& \ldots \sum_{t_{k}=0}^{t_{k-1}}\left(\begin{array}{c}
k s+t_{0} \\
(k-1) s+t_{1}
\end{array}\right) \prod_{j=0}^{k-2} a_{t_{k-j-1}-t_{k-j}}\left(\begin{array}{c}
(j-1) s+t_{k-j-1} \\
j s+t_{k-j}
\end{array}\right) . \\
& .\left(a_{t_{0}-t_{1}} \frac{x_{t_{k}+k}}{k !}+b_{t_{0}-t_{1}} \frac{x_{t_{k}+k-1}}{(k-1) !}\right), \quad(\alpha=0,1, \ldots, \sigma-s-1),
\end{aligned}
$$

où $t_{0}$ est donné par l'équation $t_{0}=\alpha-(k-2) s$ et où $k^{\prime}$ est un nombre entier positif déterminé de manière que

$$
\left.2 \leqq k^{\prime} \leqq k_{0}, \quad\left(k^{\prime}-2\right) s \leqq \alpha<\left(k^{\prime}-1\right) s .{ }^{10}\right)
$$

Démonstration. Les courbes $C_{1}$ et $C_{2}$ ayant au point $A$ un contact analytique d'ordre $s-1$, il existe un polynôme

$$
\lambda(v)=\sum_{\alpha=0}^{s-1} \frac{c_{\alpha}}{\alpha !}(v-\stackrel{\circ}{v})^{\alpha}, \quad c_{0} \neq 0,
$$

tel que par rapport à la correspondance $w=v$ on a au point $A$, qui correspond au paramètre $\stackrel{\circ}{w}=\stackrel{\circ}{v}$,

$$
y_{v}=(\lambda(v) x)_{v}, \quad v=0,1, \ldots, s-1 .
$$

8) La définition du contact analytique d'ordre $s-1$ dans un espace projectif est donné p. ex. dans [4], p. 4-5.

9) On entend par là un contact géométrique.

10) [2], p. $116\left(k_{0}=1\right)$; [3], p. $9\left(k_{0}=1\right)$; [5]; [6]. 
En vertu de

$$
\lambda(v) x(v)=\sum_{\nu=0}^{s-1} \sum_{\alpha=0}^{v}\left(\begin{array}{l}
v \\
\alpha
\end{array}\right) c_{\alpha} x_{\nu-\alpha} \frac{(v-\stackrel{o}{v})^{\nu}}{v !}+(s),
$$

on obtient d'après (3.4)

$$
y_{v}=\sum_{\alpha=0}^{v}\left(\begin{array}{l}
v \\
\alpha
\end{array}\right) c_{\alpha} x_{y-x}, \quad c_{0} \neq 0,
$$

d'où on peut calculer $c_{\alpha}(\alpha=0,1, \ldots, s-1)$.

Dans ce qui suit nous pouvons supposer, sans restreindre la généralité, que $c_{0}=1$ ainsi que (pour le cas $\left.s>1\right) c_{\alpha}=0(\alpha=1, \ldots, s-1)$, c'est-à-dire nous pouvons supposer que les équations (3.1) des courbes $C_{1}, C_{2}$ soient déterminées d'une telle manière que

$$
y_{v}=x_{v} \quad(v=0, \ldots, s-1)
$$

au point $A$ mentionné plus haut.

La condition nécessaire et suffisante pour que les courbes $C_{1}, C_{2}$ aient un contact géométrique d'ordre $s+\sigma-1$ au point $A$ est qu'il existe une correspondance analytique $w=F(v)$ telle que

$$
F(\stackrel{\circ}{v})=\stackrel{\circ}{v},\left[\frac{\mathrm{d} F(v)}{\mathrm{d} v}\right]_{v=\stackrel{\circ}{*}} \neq 0,
$$

et qu'il existe une fonction analytique $\varrho(v)$ telle que $\varrho(\stackrel{\circ}{)} \neq 0$ et telle que

$$
\left[\frac{\mathrm{d}^{v}}{\mathrm{~d} v^{\nu}} \varrho(v) x(F(v))\right]_{v=\dot{v}}=y_{v}, \quad(v=0,1, \ldots, s+\sigma-1) .
$$

On voit tout de suite qu'on a toujours

$$
\varrho(\stackrel{\circ}{v)}=1 \text {. }
$$

Le point $A$ étant un point simple de la courbe $C_{1}$, on a $\left.\left(x_{0}, x_{1}\right) \neq 0 .{ }^{11}\right)$

De cette condition et de (3.6), il résulte immédiatement qu'on obtient (3.8) et

$$
\left(\frac{\mathrm{d} F(v)}{\mathrm{d} v}\right)_{v=\dot{v}}=1, \quad\left(\frac{\mathrm{d} \varrho}{\mathrm{d} v}\right)_{v=i}=0
$$

pour $s \geqq 2$; pareillement on obtient (3.8), (3.9) et

$$
\left(\frac{\mathrm{d}^{v} F(v)}{\mathrm{d} v^{v}}\right)_{v=i}=0, \quad\left(\frac{\mathrm{d}^{v} \varrho(v)}{\mathrm{d} v^{v}}\right)_{v=i}=0, \quad(v=2,3, \ldots, s-1) \text { pour } s \geqq 3 .
$$

Or nous pouvons poser

$$
F(v)=v+\sum_{\alpha=0}^{\sigma-1} \frac{a_{\alpha}}{(s+\alpha) !}(v-\stackrel{o}{v})^{s+\alpha}+(s+\sigma)
$$

11) Le symbole $\left(x_{0}, x_{1}\right) \neq 0$ signifie que les points $x_{0}, x_{1}$ sont distincts. 
(où $a_{0} \neq-1$ dans le cas de $s=1$ ) et

$$
\varrho(v)=1+\sum_{\alpha=0}^{\sigma-1} \frac{b_{\alpha}}{(s+\alpha) !}(v-\stackrel{\circ}{v})^{s+\alpha}+(s+\sigma) .
$$

Ayant égard à l'équation [v. (2.19)]

$$
\begin{gathered}
x(F(v))=\sum_{\alpha=0}^{s+\sigma-1} \frac{x_{\alpha}}{\alpha !}(v-\stackrel{\circ}{v})^{\alpha}+\sum_{k=1}^{k_{0}} \sum_{t=(k-1) s}^{\sigma-1} \sum_{t_{1}=0}^{t_{0}} \sum_{t_{2}=0}^{t_{1}} \ldots \\
\ldots \sum_{t_{k}=0}^{t_{k-1}} \frac{x_{t_{k}+k}}{k !} \frac{(v-\stackrel{o}{v})^{s+t}}{(s+t) !} \prod_{j=0}^{k-1} A_{j}^{(k)}+(s+\sigma),
\end{gathered}
$$

où

$$
A_{j}^{(k)}=a_{t_{k-j-1}-t_{k-j}}\left(\begin{array}{c}
(k-j) s+t_{j} \\
(k-j-1) s+t_{j+1}
\end{array}\right), \quad t_{0}=t-(k-1) s
$$

et utilisant

$$
\sum_{t=0}^{s+\sigma-1} \sum_{\beta=0}^{\sigma-1} \frac{x_{t}}{t !} \frac{b_{\beta}}{(s+\beta) !}(v-\stackrel{\circ}{v})^{s+\beta+t}=\sum_{\alpha=0}^{\sigma-1} \sum_{\nu=0}^{\alpha} x_{\nu} b_{\alpha-\nu}\left(\begin{array}{c}
s+\alpha \\
v
\end{array}\right) \frac{(v-\stackrel{o}{v})^{s+\alpha}}{(s+\alpha) !}+(s+\sigma)
$$

on calcule aisément que

$$
\begin{aligned}
& \varrho(v) x(F(v))=\sum_{\alpha=0}^{s+\sigma-1} \frac{x_{\alpha}}{\alpha !}(v-v)^{\alpha}+\sum_{k=1}^{k_{n}} \sum_{t=(k-1) s}^{\sigma-1} \sum_{t_{1}=0}^{t_{n}} \sum_{t_{2}=0}^{t_{1}} \ldots \sum_{t_{k}=0}^{t_{k}} \frac{x_{t_{k}+k}}{k !} \frac{(v-\stackrel{o}{ })^{s+t}}{(s+t) !} . \\
& \cdot \prod_{j=0}^{k-1} A_{j}^{(k)}+\sum_{\alpha=0}^{\sigma-1} \sum_{v=0}^{\alpha} x_{\nu} b_{\alpha-\nu}\left(\begin{array}{c}
s+\alpha \\
v
\end{array}\right) \frac{(v-v)^{s+\alpha}}{(s+\alpha) !}+\sum_{k=1}^{k_{n}} \sum_{t=(k-1) s}^{\sigma-1} \sum_{\alpha=0}^{\sigma-1} \sum_{t_{1}=0}^{t_{n}} \sum_{t_{2}=0}^{t_{1}} \ldots \\
& \ldots \sum_{t_{k}=0}^{t_{k-1}} \frac{x_{t_{k}+k}}{k !} \frac{b_{\alpha}}{(s+\alpha) !} \frac{(v-v)^{2+\alpha+t}}{(s+t) !} \prod_{j=0}^{k-1} A_{j}^{(k)}+(s+\sigma) .
\end{aligned}
$$

Evidemment, deux cas sont à distinguer a) $k_{0}=1$, b) $k_{0}>1$.

Dans le cas où $k_{0}=1$, on peut mettre l'équation (3.10) sous la forme

$$
\begin{gathered}
\varrho(v) x(F(v))=\sum_{\alpha=0}^{s-1} \frac{x_{\alpha}}{\alpha !}(v-\grave{v})^{\alpha}+ \\
+\sum_{\alpha=0}^{\sigma-1}\left\{x_{s+\alpha}+\sum_{\nu=0}^{\alpha}\left(\begin{array}{c}
s+\alpha \\
v
\end{array}\right)\left(a_{\alpha-\nu} x_{\nu+1}+b_{\alpha-\nu} x_{\nu}\right)\right\} \frac{(v-\grave{v})^{s+\alpha}}{(s+\alpha) !}+(s+\sigma) .
\end{gathered}
$$

Le coefficient auprès de $\frac{1}{v !}(v-\stackrel{\circ}{v})^{v}, v=0,1, \ldots, s+\sigma-1$, dans la fonction (3.11) étant $\left[\frac{\mathrm{d}^{v}}{\mathrm{~d} v^{\nu}} \varrho(v) x(F(v))\right]_{v=\stackrel{\circ}{v}}$, on en déduit

$$
\begin{aligned}
{\left[\frac{\mathrm{d}^{s+\alpha}}{\mathrm{d} v^{s+\alpha}} \varrho(v) x(F(v))\right]_{v=\hat{v}} } & =x_{s+\alpha}+\sum_{\nu=0}^{\alpha}\left(\begin{array}{c}
s+\alpha \\
v
\end{array}\right)\left(a_{\alpha-\nu} x_{v+1}+b_{\alpha-\nu} x_{v}\right), \\
(\alpha & =0,1, \ldots, \sigma-1) .
\end{aligned}
$$

En substituant les expressions trouvées dans les équations (3.7) on obtient les conditions (3.2). 
Si $k_{0}>1$, nous pouvons introduire un nouveau paramètre $v$ par la relation $v=t+s+\alpha$; on déduit sans peine que

$$
\begin{aligned}
& \sum_{k=1}^{k_{0}} \sum_{t=(k-1)}^{\sigma-1} \sum_{\alpha=0}^{\sigma-1} \sum_{t_{1}=0}^{t_{0}} \sum_{t_{2}=0}^{t_{1}} \ldots \sum_{t_{k}=0}^{t_{k-1}} \frac{x_{t_{k}+k}}{k !} \frac{b_{\alpha}}{(s+\alpha) !} \frac{(v-v)^{2 s+\alpha+t}}{(s+t) !} \prod_{j=0}^{k-1} A_{j}^{(k)}= \\
&= \sum_{k=1}^{k_{0}-1} \sum_{v=\alpha+k s}^{\sigma-1} \sum_{\alpha=0}^{\sigma-k s-1} \sum_{t_{1}=0}^{t_{0}} \sum_{t_{2}=0}^{t_{1}} \ldots \sum_{t_{k}=0}^{t_{k-1}} \frac{x_{t_{k}+k}}{k !} b_{\alpha}\left(\begin{array}{l}
s+v \\
s+\alpha
\end{array}\right) \frac{(v-v)^{s+v}}{(s+v) !} \prod_{j=0}^{k-1} A_{j}^{(k)}+(s+\sigma)= \\
&= \sum_{k=1}^{k_{0}-1} \sum_{v=k s}^{\sigma-1} \sum_{\alpha=0}^{v-k s} \sum_{t_{1}=0}^{t_{0}} \sum_{t_{2}=0}^{t_{1}} \ldots \sum_{t_{k}=0}^{t_{k-1}} \frac{x_{t_{k}+k}}{k !} b_{\alpha}\left(\begin{array}{l}
s+v \\
s+\alpha
\end{array}\right) \frac{(v-\stackrel{v}{ })^{s+v}}{(s+v) !} \prod_{j=0}^{k-1} A_{j}^{(k)}+(s+\sigma),(3.12) \\
& \text { où } t_{0}=v-k s-\alpha .
\end{aligned}
$$

Faisant usage de (3.12), l'équation (3.10) prend la forme suivante

$$
\begin{aligned}
& \varrho(v) x(F(v))=\sum_{\alpha=0}^{s+\sigma-1} \frac{x_{\alpha}}{\alpha !}(v-\stackrel{\circ}{v})^{\alpha}+\sum_{\alpha=0}^{\sigma-1} \sum_{v=0}^{\alpha}\left(\begin{array}{c}
s+\alpha \\
v
\end{array}\right)\left(\alpha_{\alpha-v} x_{v+1}+b_{\alpha-v} x_{v}\right) \frac{(v-\stackrel{o}{v})^{s+\alpha}}{(s+\alpha) !}+ \\
& +\sum_{k=2}^{k_{0}} \sum_{t=(k-1)}^{\sigma-1} \sum_{t_{1}=0}^{t_{n}} \sum_{t_{2}=0}^{t_{1}} \ldots \sum_{t_{k}=0}^{t_{k-1}} \frac{x_{t_{k}+k}}{k !} \frac{(v-v)^{s+t}}{(s+t) !} \prod_{j=0}^{k-1} a_{t_{k-j-1}-t_{k-j}} . \\
& \cdot\left(\begin{array}{c}
(k-j) s+t_{j} \\
(k-j-1) s+t_{j+1}
\end{array}\right)+\sum_{x=1}^{k_{n}-1} \sum_{t=x s}^{\sigma-1} \sum_{\nu=0}^{t-\gamma s} \sum_{u_{1}=0}^{u_{0}} \sum_{u_{2}=0}^{u_{1}} \ldots \sum_{u_{x=0}}^{u_{x-1}} \frac{x_{u \nu+x}}{x !} b_{\nu} . \\
& \cdot\left(\begin{array}{l}
s+t \\
s+v
\end{array}\right) \frac{(v-\grave{v})^{s+t}}{(s+t) !} \prod_{j=0}^{x-1} a_{u_{\varkappa-j-1}-u_{\varkappa-j}}\left(\begin{array}{c}
(\varkappa-j) s+u_{j} \\
(\varkappa-j-1) s+u_{j+1}
\end{array}\right)+(s+\sigma),
\end{aligned}
$$

où

$$
\text { a) } t_{0}=t-(k-1) s \text {, b) } u_{0}=t-x s-v \text {. }
$$

Introduisons maintenant dans (3.13) au lieu du paramètre $v$ un nouveau paramètre $u_{0}$ moyennant l'équation $(3.14 \mathrm{~b})$, au lieu du paramètre $t$ un nouveau paramètre $\alpha$ tel que $\alpha=t-s$ et posons enfin

$$
\varkappa=k-1, u_{i}=t_{i+1}(i=0, \ldots, k-1) .
$$

Ceci étant, l'équation (3.13) devient

$$
\begin{aligned}
& \varrho(v) x(F(v))=\sum_{\alpha=0}^{s+\sigma-1} \frac{x_{\alpha}}{\alpha !}(v-\stackrel{o}{v})^{\alpha}+\sum_{\alpha=0}^{\sigma-1} \sum_{v=0}^{\alpha}\left(\begin{array}{c}
s+\alpha \\
v
\end{array}\right)\left(a_{\alpha-v} x_{v+1}+b_{\alpha-v} x_{v}\right) \frac{(v-\stackrel{o}{v})^{s+\alpha}}{(s+\alpha) !}+ \\
& +\sum_{k=2}^{k_{0}} \sum_{\alpha=(k-2) s}^{\sigma-1-s} \sum_{t_{1}=0}^{t_{0}} \sum_{t_{2}=0}^{t_{1}} \ldots \sum_{t_{k}=0}^{t_{k-1}} \frac{x_{t_{k}+k}}{k !} a_{t_{0}-t_{1}}\left(\begin{array}{c}
k s+t_{0} \\
\left.(k-1) s+t_{1}\right)
\end{array}\right) \prod_{j=0}^{k-2} a_{t_{k-j-1}-t_{k-j}} .
\end{aligned}
$$

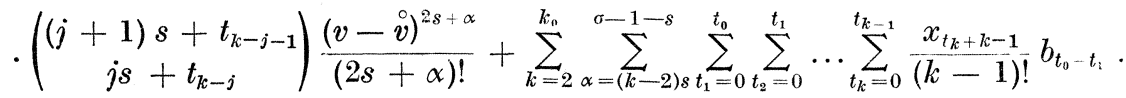

$$
\begin{aligned}
& \cdot\left(\begin{array}{c}
k s+t_{0} \\
(k-1) s+t_{1}
\end{array}\right) \prod_{j=0}^{k-2} a_{t_{k-j-1}-t_{k-j}}\left(\begin{array}{c}
(j+1) s+t_{k-j-1} \\
j s+t_{k-j}
\end{array}\right) \frac{(v-i o)^{2 s+\alpha}}{(2 s+\alpha) !}+(s+\sigma),
\end{aligned}
$$


où $t_{0}=\alpha-(k-2) s$, ce qu'on peut mettre, en définitive, sous la forme

$$
\begin{aligned}
& \varrho(v) x(F(v))=\sum_{\alpha=0}^{s-1} \frac{x_{\alpha}}{\alpha !}(v-\stackrel{\circ}{\alpha})^{\alpha}+\sum_{\alpha=0}^{s-1}\left\{x_{s+\alpha}+\sum_{\nu=0}^{\alpha}\left(\begin{array}{c}
s+\alpha \\
v
\end{array}\right)\left(a_{\alpha-\nu} x_{v+1}+b_{\alpha-\nu} x_{v}\right)\right\} .
\end{aligned}
$$

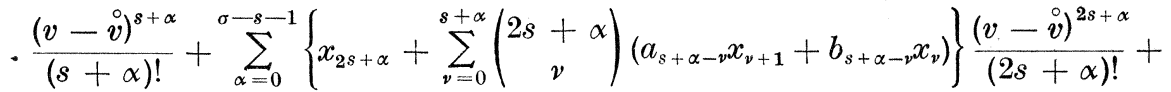

$$
\begin{aligned}
& +\sum_{k=2}^{k_{0}} \sum_{\alpha=(k-2) s}^{\sigma-s-1} \sum_{t_{1}=0}^{t_{0}} \sum_{t_{2}=0}^{t_{0}} \ldots \sum_{t_{k}=0}^{t_{k-1}}\left(\begin{array}{c}
k s+t_{0} \\
(k-1) s+t_{1}
\end{array}\right) \prod_{j=0}^{k-2} a_{t_{k-j-1}-t_{k-j}} . \\
& \text {. }\left(\begin{array}{c}
(j+1) s+t_{k-j-1} \\
j s+t_{k-j}
\end{array}\right)\left(a_{t_{0}-t_{1}} \frac{x_{t_{k}+k}}{k !}+b_{t_{0}-t_{1}} \frac{x_{t_{k}+k-1}}{(k-1) !}\right) \frac{(v-\stackrel{o}{ })^{2 s+\alpha}}{(2 s+\alpha) !}+(s+\sigma) \text {. }
\end{aligned}
$$

Il en résulte

$$
\begin{gathered}
{\left[\frac{\mathrm{d}^{s+\alpha} \varrho(v) x(F(v))}{\mathrm{d} v^{s+\alpha}}\right]_{v=v}=x_{s+\alpha}+\sum_{v=0}^{\alpha}\left(\begin{array}{c}
s+\alpha \\
v
\end{array}\right)\left(a_{\alpha-v} x_{v+1}+b_{\alpha-v} x_{v}\right),} \\
(\alpha=0,1, \ldots, s-1) ; \\
{\left[\frac{\mathrm{d}^{2 s+\alpha} \varrho(v) x(F(v))}{\mathrm{d} v^{2 s+\alpha}}\right]_{v=i}=x_{2 s+\alpha}+\sum_{v=0}^{s+\alpha}\left(\begin{array}{c}
2 s+\alpha \\
v
\end{array}\right)\left(a_{s+\alpha-v} x_{v+1}+b_{s+\alpha-\nu} x_{v}\right)+} \\
+\sum_{k=2}^{k^{\prime}} \sum_{t_{1}=0}^{t_{0}} \sum_{t_{2}=0}^{t_{1}} \ldots \sum_{t_{k}=0}^{t_{k+1}}\left(\begin{array}{c}
k s+t_{0} \\
(k-1) s+t_{1}
\end{array}\right) \prod_{j=0}^{k-2} a_{t_{k-j-1}-t_{k-j}}\left(\begin{array}{c}
(j+1) s+t_{k-j-1} \\
j s+t_{k-j}
\end{array}\right) . \\
\cdot\left(a_{t_{0}-t_{1}} \frac{x_{t_{k}+k}}{k !}+b_{t_{0}-t_{1}} \frac{x_{t_{k+k-1}}}{(k-1) !}\right), \quad(\alpha=0,1, \ldots, \sigma-s-1),
\end{gathered}
$$

où $k^{\prime}$ est un nombre entier positif tel que $2 \leqq k^{\prime} \leqq k_{0},\left(k^{\prime}-2\right) s \leqq \alpha<$ $<\left(k^{\prime}-1\right) . s$.

On en déduit sans difficulté (3.3).

4. Simplification des conditions fondamentales du contact des courbes dans l'espace projectif. Les conditions trouvées (3.2) et (3.3) du contact des courbes dans l'espace projectif sont linéaires en $b_{v}$. Quant aux coefficients $a_{v}$, les conditions trouvées sont linéaires seulement dans le cas où $k_{0}=1$; en supposant $k_{0}>1$, on voit sans peine que parmi les $\sigma$ conditions (3.2) et (3.3) exactement $s$ sont linéaires, $s$ quadratiques, .., $s$ de degré $k_{0}-1$ et $\sigma-\left(k_{0}-1\right) s$ de degré $k_{0}$ en $a_{v}$. C'est pourquoi l'application du Théorème 2 aux problèmes généraux d'augmentation du contact de deux courbes données par projection et d'étude du contact des projections d'une courbe de deux centres différents est très difficile.

Pour résoudre quelques problèmes particuliers relatifs au contact des courbes, il n'est pas nécessaire de faire usage des conditions (3.2) et (3.3) en forme explicite; il suffit de connaître la distribution des nombres $a_{v}, b_{v}(v=0, \ldots$, $\ldots, \sigma-1)$ dans les coefficients de $\dot{x}_{\mu}(\mu=0, \ldots, s+\alpha+1 ; \alpha=0,1, \ldots$, $\ldots, \sigma-s-1$ ) dans les conditions (3.2) et (3.3).

De ce point de vue, nous allons simplifier les conditions (3.2) et (3.3); nous distinguerons deux cas: a) $s=1$, b) $s>1$. 
I. Soit $s=1$. En transformant les conditions (3.2) et (3.3), on voit sans peine que dans ce cas nous pouvons énoncer le Théorème 2 sous la forme suivante:

Théorème 3. Soit $\sigma \geqq 1$ un nombre entier. Supposons que les courbes $C_{1}$ et $C_{2}$ aient au point $A$ un contact analytique d'ordre 0 . Soit $y_{0}=x_{0}$.

Les courbes $C_{1}, C_{2}$ ont au point $A$ un contact d'ordre $\sigma$ justement lorsqu'on peut trouver des nombres $a_{v}, b_{v}\left(v=0, \ldots, \sigma-1 ; a_{0} \neq-1\right)$ tels que

$$
y_{1}-x_{1}=a_{0} x_{1}+b_{0} x_{0}
$$

ainsi que (en supposant $\sigma>1$ )

$$
\begin{gathered}
y_{1+\alpha}-x_{1+\alpha}=\sum_{\nu=0}^{\alpha}\left(\begin{array}{c}
1+\alpha \\
v
\end{array}\right)\left(a_{\alpha-\nu} x_{v+1}+b_{\alpha-\nu} x_{\nu}\right)+ \\
+\sum_{k=2}^{1+\alpha} \sum_{t_{1}=0}^{1+\alpha+k} \sum_{t_{2}=0}^{t_{1}} \ldots \sum_{t_{k}=0}^{t_{k-1}}\left(\begin{array}{c}
1+\alpha \\
k-1+t_{1}
\end{array}\right)\left(\begin{array}{l}
k-1+t_{1} \\
k-2+t_{2}
\end{array}\right) \ldots\left(\begin{array}{l}
2+t_{k-2} \\
1+t_{k-1}
\end{array}\right)\left(\begin{array}{c}
1+t_{k-1} \\
t_{k}
\end{array}\right) . \\
. a_{t_{k-1}-t_{k}} a_{t_{k-2}-t_{k-1}} \ldots a_{t_{1}-t_{2}}\left(\frac{1}{k !} a_{1+\alpha-k-t_{1}} x_{t_{k+k}}+\frac{1}{(k-1) !} b_{1+\alpha-k-t_{1}} x_{t_{k+k-1}}\right), \\
(\alpha=1, \ldots, \sigma-1) .
\end{gathered}
$$

En étudiant les questions concernant le contact des projections des courbes, supposé que les courbes $C_{1}, C_{2}$ données aient un point commun $(s=1)$, on ne peut pas appliquer du tout les résultats trouvés. Dans le Théorème 2 (et par conséquence de même dans l'énoncé précédent) nous avons supposé que $y_{0}=$ $=x_{0}$. En continuant à supposer que les courbes $C_{1}, C_{2}$ aient un contact analytique d'ordre 0 au point commun $A$, il faut encore prendre en considération le cas où $x_{0}, y_{0}$ satisfont seulement une équation de la forme [v. (3.5)]

$$
b x_{0}=y_{0}, \quad b \neq 0 .
$$

Nous pouvons donc énoncer le théorème suivant:

Théorème 4. Soit $\sigma \geqq 1$ un nombre entier. Supposons que les courbes $C_{1}, C_{2}$ aient au point commun $A$ un contact analytique d'ordre 0 . Soit $b x_{0}=y_{0}(b \neq 0)$.

Les courbes $C_{1}, C_{2}$ ont au point $A$ un contact d'ordre $\sigma$ justement lorsqu'on peut trouver des nombres $a_{v}, b_{v}\left(v=0, \ldots, \sigma-1 ; a_{0} \neq-1\right)$ tels que

$$
y_{1}-b x_{1}=a_{0} b x_{1}+b_{0} x_{0}
$$

ainsi que (en supposant $\sigma>1$ )

$$
\begin{aligned}
& y_{1+\alpha}-b x_{1+\alpha}=\sum_{\nu=0}^{\alpha}\left(\begin{array}{c}
1+\alpha \\
v
\end{array}\right)\left(b a_{\alpha-\nu} x_{\nu+1}+b_{\alpha-\nu} x_{\nu}\right)+\sum_{k=2}^{1+\alpha} \sum_{t_{1}=0}^{1+\alpha-k} \sum_{t_{2}=0}^{t_{1}} \ldots \\
& \ldots \sum_{t_{k}=0}^{t_{k-1}}\left(\begin{array}{c}
1+\alpha \\
k-1+t_{1}
\end{array}\right)\left(\begin{array}{c}
k-1+t_{1} \\
k-2+t_{2}
\end{array}\right) \ldots\left(\begin{array}{c}
2+t_{k-2} \\
1+t_{k-1}
\end{array}\right)\left(\begin{array}{c}
1+t_{k-1} \\
t_{k}
\end{array}\right) a_{t_{k-1}-t_{k}} a_{t_{k-2}-t_{k-1}} \ldots \\
& \ldots a_{t_{1}-t_{2}}\left(\frac{1}{k !} b a_{1+\alpha-k-t_{1}} x_{t_{k}+k}+\frac{1}{(k-1) !} b_{1+\alpha-k-t_{1}} x_{t_{k}+k-1}\right), \quad(\alpha=1,2, \ldots, \sigma-1) .
\end{aligned}
$$


Démonstration. En remplaçant les équations paramétriques (3.1a) de la courbe $C_{1}$, c'est-à-dire les équations $C_{1} \equiv x=x(w)$, par les équations

$$
C_{1} \equiv \tilde{x}=b x(w), \quad b \neq 0,
$$

on obtient, d'après (4.5), $\tilde{x}_{0}=y_{0}$.

Pour les courbes $C_{1}, C_{2}$ données respectivement par les équations (4.6) et (3.1b), on peut donc faire usage du Théorème 3 où l'on pose $b^{-1} b_{v}$ au lieu de $b_{v}(v=0, \ldots, \sigma-1)$; on en déduit sans difficulté (4.4) et (4.5).

Les équations (4.1) et (4.2) n'étant qu'un cas particulier des équations (4.4) et (4.5) respectivement pour $b=1$, il suffit de traiter les coefficients de $x_{\mu}$ $(\mu=0, \ldots, 1+\alpha ; \alpha=1, \ldots, \sigma-1)$ en (4.5) seulement.

Lemme 1. Supposons que l'équation

$$
y_{1+\alpha}-b x_{1+\alpha}=\sum_{\mu=0}^{1+\alpha} L_{1, \alpha}^{\mu} x_{\mu}
$$

équivaille à l'équation (4.4) pour $\alpha=0$ et à l'équation (4.5) pour $\alpha>0$.

Alors on a

a) $L_{1, \alpha}^{0}=b_{\alpha}$,

b) $L_{1, \alpha}^{\mu}=\left(\begin{array}{c}1+\alpha \\ \mu-1\end{array}\right) b a_{1+\alpha-\mu}\left(1+a_{0}\right)^{\mu-1}+\left(\begin{array}{c}1+\alpha \\ \mu\end{array}\right) b_{\alpha-\mu}\left(1+a_{0}\right)^{\mu}+* L_{1, \alpha}^{\mu}$,

$$
(0<\alpha ; \mu=1, \ldots, \alpha) \text {, }
$$

c) $L_{1, \alpha}^{\alpha+1}=b\left[\left(1+a_{0}\right)^{\alpha+1}-1\right]$,

ò̀ $* L_{1, \alpha}^{\mu}(\mu<\alpha)$ est un polynôme en $a_{v}(v=0, \ldots, \alpha-\mu), b_{\lambda}(\lambda=0, \ldots$, $\ldots, \alpha-1-\mu), b ;{ }^{*} L_{1, \alpha}^{\alpha}=0$.

Démonstration. En supposant $\alpha=1$, on trouve de (4.5)

$$
y_{2}-b x_{2}=b_{1} x_{0}+\left[b a_{1}+2 b_{0}\left(1+a_{0}\right)\right] x_{1}+b\left[\left(1+a_{0}\right)^{2}-1\right] x_{2} ;
$$

en supposant $\alpha>1$ :

$$
\begin{aligned}
& y_{1+\alpha}-b x_{1+\alpha}=b \sum_{\nu=0}^{\alpha}\left(\begin{array}{c}
1+\alpha \\
v
\end{array}\right) a_{\alpha-\nu} x_{\nu+1}+\sum_{\nu=0}^{\alpha}\left(\begin{array}{c}
1+\alpha \\
v
\end{array}\right) b_{\alpha-\nu} x_{v}+ \\
& +\frac{b}{2} \sum_{t_{1}=0}^{\alpha-1} \sum_{t_{2}=0}^{t_{1}}\left(\begin{array}{c}
1+\alpha \\
1+t_{1}
\end{array}\right)\left(\begin{array}{c}
1+t_{1} \\
t_{2}
\end{array}\right) a_{t_{1}-t_{2}} a_{\alpha-1-t} x_{t_{2}+2}+\sum_{t_{1}=0}^{\alpha-1} \sum_{t_{2}=0}^{t_{1}}\left(\begin{array}{c}
1+\alpha \\
1+t_{1}
\end{array}\right)\left(\begin{array}{c}
1+t_{1} \\
t_{2}
\end{array}\right) . \\
& \text {. } a_{t_{1}-t_{2}} b_{\alpha-1-t_{1}} x_{t_{2}+1}+b \sum_{k=3}^{1+\alpha} \sum_{t_{k}=0}^{1+\alpha-k} \sum_{t_{k-1}=t_{k}}^{1+\alpha-k} \ldots \sum_{t_{1}=t_{2}}^{1+\alpha-k} \frac{1}{k !}\left(\begin{array}{c}
1+t_{k-1} \\
t_{k}
\end{array}\right)\left(\begin{array}{l}
2+t_{k-2} \\
1+t_{k-1}
\end{array}\right) \cdots \\
& \ldots\left(\begin{array}{l}
k-1+t_{1} \\
k-2+t_{2}
\end{array}\right)\left(\begin{array}{c}
1+\alpha \\
k-1+t_{1}
\end{array}\right) a_{t_{k-1}-t_{k}} \ldots a_{t_{1}-t_{2}} a_{1+\alpha-k-t_{1}} x_{t_{k}+k}+ \\
& +\sum_{k=3}^{1+\alpha} \sum_{t_{k}=0}^{1+\alpha-k} \sum_{t_{k-1}=t_{k}}^{1+\alpha-k} \ldots \sum_{t_{1}=t_{2}}^{1+\alpha-k} \frac{1}{(k-1) !}\left(\begin{array}{c}
1+t_{k-1} \\
t_{k}
\end{array}\right)\left(\begin{array}{c}
2+t_{k-2} \\
1+t_{k-1}
\end{array}\right) \ldots \\
& \cdots\left(\begin{array}{l}
k-1+t_{1} \\
k-2+t_{2}
\end{array}\right)\left(\begin{array}{c}
1+\alpha \\
k-1+t_{1}
\end{array}\right) a_{t_{k-1}-t_{k}} \ldots a_{t_{1}-t_{2}} b_{1+\alpha-k-t_{1}} x_{t_{k}+k-1} .
\end{aligned}
$$


En introduisant un nouveau paramètre auxiliaire $\mu$, on en déduit sans peine

$$
\begin{aligned}
& y_{1+\alpha}-b x_{1+\alpha}=b \sum_{\mu=1}^{1+\alpha}\left(\begin{array}{c}
1+\alpha \\
\mu-1
\end{array}\right) a_{1+\alpha-\mu} x_{\mu}+\sum_{\mu=0}^{\alpha}\left(\begin{array}{c}
1+\alpha \\
\mu
\end{array}\right) b_{\alpha-\mu} x_{\mu}+ \\
& +\frac{b}{2} \sum_{\mu=2}^{1+\alpha} \sum_{\nu=\mu-2}^{\alpha-1}\left(\begin{array}{l}
1+\alpha \\
1+v
\end{array}\right)\left(\begin{array}{l}
1+\nu \\
\mu-2
\end{array}\right) a_{2+\nu-\mu} a_{\alpha-1-\nu} x_{\mu}+\sum_{\mu=1}^{\alpha} \sum_{\nu=\mu-1}^{\alpha-1}\left(\begin{array}{l}
1+\alpha \\
1+v
\end{array}\right)\left(\begin{array}{l}
1+v \\
\mu-1
\end{array}\right) . \\
& \text {. } a_{1+\nu-\mu} b_{\alpha-1-\nu} x_{\mu}+b \sum_{k=3}^{1+\alpha} \sum_{\mu=k}^{1+\alpha} \sum_{t_{k-1}=\mu-k}^{1+\alpha-k} \sum_{t_{k-2}=t_{k-1}}^{1+\alpha-k} \ldots \sum_{t_{1}=t_{2}}^{1+\alpha-k} \frac{1}{k !}\left(\begin{array}{c}
1+t_{k-1} \\
\mu-k
\end{array}\right)\left(\begin{array}{c}
2+t_{k-2} \\
1+t_{k-1}
\end{array}\right) \ldots \\
& \ldots\left(\begin{array}{l}
k-1+t_{1} \\
k-2+t_{2}
\end{array}\right)\left(\begin{array}{c}
1+\alpha \\
k-1+t_{1}
\end{array}\right) a_{t_{k-1}+k-\mu} a_{t_{k-2}-t_{k-1}} \ldots a_{t_{1}-t_{2}} a_{1+\alpha-k-t_{1}} x_{\mu}+ \\
& +\sum_{k=3}^{1+\alpha} \sum_{\mu=k-1}^{\alpha} \sum_{t_{k-1}=\mu-k+1}^{1+\alpha-k} \sum_{t_{k-2}=t_{k-1}}^{1+\alpha-k} \ldots \sum_{t_{1}=t_{2}}^{1+\alpha-k} \frac{1}{(k-1) !}\left(\begin{array}{c}
1+t_{k-1} \\
\mu-k+1
\end{array}\right)\left(\begin{array}{l}
2+t_{k-2} \\
1+t_{k-1}
\end{array}\right) \ldots \\
& \ldots\left(\begin{array}{l}
k-1+t_{1} \\
k-2+t_{2}
\end{array}\right)\left(\begin{array}{c}
1+\alpha \\
k-1+t_{1}
\end{array}\right) a_{t_{k-1}+k-1-\mu} a_{t_{k-2}-t_{k-1}} \ldots a_{t_{1}-t_{2}} b_{1+\alpha-k-t_{1}} x_{\mu} .
\end{aligned}
$$

En comparant respectivement (4.4), (4.9) et (4.10) avec (4.7), on obtient successivement pour $L_{1, \alpha}^{\mu}(\mu=0, \ldots, 1+\alpha ; \alpha \geqq 0)$ les conditions cherchées.

a) Avant tout on voit sans peine de (4.4), (4.9) et (4.10) que $L_{1,0}^{0}=b_{0}$, $L_{1,1}^{0}=b_{1}, L_{1, \alpha}^{0}=\left(\begin{array}{c}1+\alpha \\ 0\end{array}\right) b_{\alpha},(\alpha>1)$, alors l'équation (4.8a) est valable.

b) Pour $L_{1, \alpha}^{\mu}(0<\alpha ; \mu=1, \ldots, \alpha)$ nous trouvons de (4.9)

$$
L_{1,1}^{1}=b a_{1}+2 b_{0}\left(1+a_{0}\right) \text {. }
$$

En supposant $\alpha>1$, on obtient de (4.10) d'une part

$$
L_{1, \alpha}^{1}=b a_{\alpha}+(1+\alpha) b_{\alpha-1}\left(1+a_{0}\right)+\sum_{\nu=1}^{\alpha-1}\left(\begin{array}{l}
1+\alpha \\
1+v
\end{array}\right) a_{v} b_{\alpha-1-\nu},
$$

d'autre part

$$
L_{1,2}^{2}=3 b a_{1}\left(1+a_{0}\right)+3 b_{0}\left(1+a_{0}\right)^{2} ;
$$

en supposant $\alpha>2$, on obtient de (4.10) de même

$$
L_{1, \alpha}^{2}=(1+\alpha) b a_{\alpha-1}\left(1+a_{0}\right)+\left(\begin{array}{c}
1+\alpha \\
2
\end{array}\right) b_{\alpha-2}\left(1+a_{0}\right)^{2}+* L_{1, \alpha}^{2}
$$

où $* L_{1, \alpha}^{2}$ est un polynôme du 3 e degré en $b, a_{0}, a_{1}, \ldots, a_{\alpha-2}, b_{0}, \ldots, b_{\alpha-3}$.

Enfin, en supposant $\alpha>2$ et $\mu>2$, il résulte de (4.10)

$$
\begin{gathered}
L_{1, \alpha}^{\mu}=b\left(\begin{array}{c}
1+\alpha \\
\mu-1
\end{array}\right) a_{1+\alpha-\mu}+\left(\begin{array}{c}
1+\alpha \\
\mu
\end{array}\right) b_{\alpha-\mu}+\frac{b}{2} \sum_{\nu=\mu-2}^{\alpha-1}\left(\begin{array}{l}
1+\alpha \\
1+\nu
\end{array}\right)\left(\begin{array}{l}
1+\nu \\
\mu-2
\end{array}\right) . \\
. a_{2+\nu-\mu} a_{\alpha-1-\nu}+\sum_{\nu=\mu-1}^{\alpha-1}\left(\begin{array}{l}
1+\alpha \\
1+\nu
\end{array}\right)\left(\begin{array}{c}
1+\nu \\
\mu-1
\end{array}\right) a_{1+\nu-\mu} b_{\alpha-1-\nu}+b \sum_{k=3}^{\mu} \sum_{t_{k-1}=\mu-k t_{k-2}}^{1+\alpha-k} \sum_{k-1}^{1+\alpha-k} \ldots
\end{gathered}
$$




$$
\begin{gathered}
\ldots \sum_{t_{1}=t_{2}}^{1+\alpha-k} \frac{1}{k !}\left(\begin{array}{c}
1+t_{k-1} \\
\mu-k
\end{array}\right)\left(\begin{array}{l}
2+t_{k-2} \\
1+t_{k-1}
\end{array}\right) \ldots\left(\begin{array}{l}
k-1+t_{1} \\
k-2+t_{2}
\end{array}\right)\left(\begin{array}{c}
1+\alpha \\
k-1+t_{1}
\end{array}\right) . \\
. a_{t_{k-1}+k-\mu} a_{t_{k-2}-t_{k-1}} \ldots a_{t_{1}-t_{2}} a_{1+\alpha-k-t_{1}}+\sum_{k=3}^{\mu+1} \sum_{t_{k-1}=\mu-k+1}^{1+\alpha-k} \sum_{t_{k-2}=t_{k-1}}^{1+\alpha-k} \ldots \\
\ldots \sum_{t_{1}=t_{2}}^{1+\alpha-k} \frac{1}{(k-1) !}\left(\begin{array}{c}
1+t_{k-1} \\
\mu-k+1
\end{array}\right)\left(\begin{array}{l}
2+t_{k-2} \\
1+t_{k-1}
\end{array}\right) \ldots\left(\begin{array}{l}
k-1+t_{1} \\
k-2+t_{2}
\end{array}\right)\left(\begin{array}{c}
1+\alpha \\
k-1+t_{1}
\end{array}\right) . \\
. a_{t_{k-1}+k-1-\mu} a_{t_{k-2}-t_{k-1}} \ldots a_{t_{1}-t_{2}} b_{1+\alpha-k+t_{1}} .
\end{gathered}
$$

On voit immédiatement, que, pour les nombres $a_{v}, b_{\lambda}$ dans $L_{1, \alpha}^{\mu}(\mu>2$, $\alpha>2$ étant fixes), on a $v_{\max }=1+\alpha-\mu ; \lambda_{\max }=\alpha-\mu$.

Le produit

$$
a_{t_{k-1}+k-\mu} a_{t_{k-2}-t_{k-1}} \ldots a_{t_{1}-t_{2}} a_{1+\alpha-k-t_{1}}
$$

$\left(k=3, \ldots, \mu ; t_{k-1}=\mu-k, \ldots, 1+\alpha-k ; t_{i}=t_{i+1}, \ldots, 1+\alpha-k ; i=1, \ldots\right.$, $\ldots, k-2)$ contient le facteur $a_{1+\alpha-\mu}$ alors et alors seulement si l'on est dans un des cas suivants
а) $t_{k-1}=t_{k-2}=\ldots=t_{1}=1+\alpha-k$,
в) $t_{k-1}=\ldots=t_{k-i}=\mu-k ; t_{k-i-1}=\ldots=t_{1}=1+\alpha-k(i=1, \ldots$, $\ldots, k-2)$,
r) $t_{k-1}=t_{k-2}=\ldots=t_{1}=\mu-k$.

En outre, on voit sans peine que le facteur $\alpha_{1+\alpha-\mu}$ ne se trouve pas dans les membres de l'expression $L_{1, \alpha}^{\mu}(\alpha>2, \mu>2)$ qui contiennent $b_{\nu}(v=0, \ldots$, $\ldots, \alpha-\mu)$ comme un facteur.

Cela étant, pour la somme de tous les membres de l'expression $L_{1, \alpha}^{\mu}(\alpha>2$, $\mu>2$ ) contenants le facteur $\alpha_{1+\alpha-\mu}$, nous trouvons

$$
\begin{gathered}
b a_{1+\alpha-\mu}\left\{\left(\begin{array}{l}
1+\alpha \\
\mu-1
\end{array}\right)+\left(\begin{array}{c}
1+\alpha \\
\mu-1
\end{array}\right)(\mu-1) a_{0}+\sum_{k=3}^{\mu} \frac{1}{k !}\left[\left(\begin{array}{c}
2-k+\alpha \\
\mu-k
\end{array}\right)\left(\begin{array}{l}
3-k+\alpha \\
2-k+\alpha
\end{array}\right) \ldots\right.\right. \\
\ldots\left(\begin{array}{c}
\alpha \\
\alpha-1
\end{array}\right)\left(\begin{array}{c}
1+\alpha \\
\alpha
\end{array}\right)+\sum_{i=1}^{k-2}\left(\begin{array}{c}
1+\mu-k \\
\mu-k
\end{array}\right)\left(\begin{array}{l}
2+\mu-k \\
1+\mu-k
\end{array}\right) \ldots\left(\begin{array}{c}
i+\mu-k \\
i-1+\mu-k
\end{array}\right) . \\
\cdot\left(\begin{array}{c}
i+2-k+\alpha \\
i+\mu-k
\end{array}\right)\left(\begin{array}{l}
i+3-k+\alpha \\
i+2-k+\alpha
\end{array}\right) \ldots\left(\begin{array}{c}
\alpha \\
\alpha-1
\end{array}\right)\left(\begin{array}{c}
1+\alpha \\
\alpha
\end{array}\right)+ \\
\left.\left.+\left(\begin{array}{c}
1+\mu-k \\
\mu-k
\end{array}\right)\left(\begin{array}{l}
2+\mu-k \\
1+\mu-k
\end{array}\right) \ldots\left(\begin{array}{l}
\mu-1 \\
\mu-2
\end{array}\right)\left(\begin{array}{c}
1+\alpha \\
\mu-1
\end{array}\right)\right] a_{0}^{k-1}\right\}= \\
=b a_{1+\alpha-\mu}\left\{\left(\begin{array}{c}
1+\alpha \\
\mu-1
\end{array}\right)+\left(\begin{array}{c}
1+\alpha \\
\mu-1
\end{array}\right)(\mu-1) a_{0}+\sum_{k=3}^{\mu}\left(\begin{array}{l}
1+\alpha \\
\mu-1
\end{array}\right)\left(\begin{array}{l}
\mu-1 \\
k-1
\end{array}\right) a_{0}^{k-1}\right\},
\end{gathered}
$$

c'est ce qu'on peut mettre sous la forme définitive

$$
b a_{1+\alpha-\mu}\left(\begin{array}{l}
1+\alpha \\
\mu-1
\end{array}\right)\left(1+a_{0}\right)^{\mu-1} .
$$


Pareillement, pour la somme de tous les membres de $L_{1, \alpha}^{\mu}(\alpha>2, \mu>2)$ contenant le facteur $b_{\alpha-\mu}$, on obtient

$$
b_{\alpha-\mu}\left(\begin{array}{c}
1+\alpha \\
\mu
\end{array}\right)\left(1+a_{0}\right)^{\mu} .
$$

De $(4.15)-(4.17)$ il résulte qu'on peut écrire $L_{1, \alpha}^{\mu}(\alpha>2, \mu>2)$ comme suit $L_{1, \alpha}^{\mu}=b a_{1+\alpha-\mu}\left(\begin{array}{c}1+\alpha \\ \mu-1\end{array}\right)\left(1+a_{0}\right)^{\mu-1}+b_{\alpha-\mu}\left(\begin{array}{c}1+\alpha \\ \mu\end{array}\right)\left(1+a_{0}\right)^{\mu}+* L_{1, \alpha}^{\mu}$, où $* L_{1, \alpha}^{\mu}$ est un polynôme en $b, a_{v}(\nu=0, \ldots, \alpha-\mu), b_{\lambda}(\lambda=0, \ldots, \alpha-1-\mu)$.

De (4.15) on voit sans difficulté que dans le cas où $\mu=\alpha$ et dans ce cas seulement l'ensemble de tous les membres de (4.15) est donné par la somme des membres (4.16) et (4.17). Des relations $(4.11)-(4.14)$ et (4.18) il résulte ensuite (4.8b).

c) Enfin, de (4.9) et (4.10) (en supposant $\alpha>1$ ) on déduit respectivement et

$$
L_{1,1}^{2}=b\left[\left(1+a_{0}\right)^{2}-1\right]
$$

$$
\begin{gathered}
L_{1, \alpha}^{1+\alpha}=b\left(\begin{array}{c}
1+\alpha \\
\alpha
\end{array}\right) a_{0}+\frac{b}{2}\left(\begin{array}{c}
1+\alpha \\
\alpha
\end{array}\right)\left(\begin{array}{c}
\alpha \\
\alpha-1
\end{array}\right) a_{0}^{2}+b \sum_{k=3}^{1+\alpha} \frac{1}{k !}\left(\begin{array}{c}
2+\alpha-k \\
1+\alpha-k
\end{array}\right) . \\
\cdot\left(\begin{array}{c}
3+\alpha-k \\
2+\alpha-k
\end{array}\right) \ldots\left(\begin{array}{c}
\alpha \\
\alpha-1
\end{array}\right)\left(\begin{array}{c}
1+\alpha \\
\alpha
\end{array}\right) a_{0}^{k}=b\left[\left(1+a_{0}\right)^{1+\alpha}-1\right] .
\end{gathered}
$$

Les équations (4.19) et (4.20) avec la relation $L_{1,0}^{1}=b a_{0}$, qui est une conséquence de (4.4), démontrent les équations $(4.8 \mathrm{c})$.

II. Soit $s>1$. En supposant respectivement $k_{0}=1, k_{0}>1$, il faut, dans ce cas, simplifier les équations (3.2) ainsi que les équations (3.3a,b).

Lemme 2. Soit $s>1$. Supposons que les équations

$$
y_{s+\alpha}-x_{s+\alpha}=\sum_{\mu=0}^{1+\alpha} L_{s, \alpha}^{\mu} x_{\mu}
$$

équivaillent, pour $0 \leqq \alpha<s$, respectivement aux équations (3.2) et (3.3a), et $q u$ 'elles équivaillent, pour $s \leqq \alpha$, aux équations $(3.3 \mathrm{~b}) .{ }^{12}$ )

Ensuite on a

$$
\begin{aligned}
& \text { a) } L_{s, \alpha}^{0}=b_{\alpha} \\
& \text { b) } L_{s, \alpha}^{\mu}=\left(\begin{array}{c}
s+\alpha \\
\mu-1
\end{array}\right) a_{1+\alpha-\mu}+\left(\begin{array}{c}
s+\alpha \\
\mu
\end{array}\right) b_{\alpha-\mu}+* L_{s, \alpha}^{\mu} \\
& \quad(0<\alpha ; \mu=1, \ldots, \alpha) \\
& \text { c) } L_{s, \alpha}^{1+\alpha}=\left(\begin{array}{c}
s+\alpha \\
s
\end{array}\right) a_{0}
\end{aligned}
$$

12) Plus précisément: que l'équation (4.21) équivaille, pour $\alpha=s+\alpha^{\prime}$, à l'équation (3.3b) pour $\alpha^{\prime}$. 
ò̀ $* L_{s, \alpha}^{\mu}(s \leqq \alpha, \mu<\alpha)$ est un polynôme en $a_{v}(v=0, \ldots, \alpha-\mu), b_{\lambda}(\lambda=0, \ldots$, $\ldots, \alpha-1-\mu)$ et où

$$
\begin{aligned}
& \text { a) } * L_{s, \alpha}^{\mu}=0,(\alpha=1, \ldots, s-1 ; \mu=1, \ldots, \alpha), \\
& \text { b) } * L_{2, \alpha}^{\alpha}=3\left(\begin{array}{c}
2+\alpha \\
4
\end{array}\right) a_{0}^{2},(\alpha \geqq 2), \\
& \text { c) } * L_{s, \alpha}^{\alpha}=0,(s>2 ; \alpha \geqq s) .
\end{aligned}
$$

Démonstration. On voit tout de suite qu'on peut prendre respectivement les équations (3.2) et (3.3a) sous la forme suivante $(0 \leqq \alpha<s)$

a) $y_{s}-x_{s}=b_{0} x_{0}+a_{0} x_{1}$,

b) $y_{s+\alpha}-x_{s+\alpha}=b_{\alpha} x_{0}+\sum_{\mu=1}^{\alpha}\left\{\left(\begin{array}{c}s+\alpha \\ \mu-1\end{array}\right) a_{\alpha+1-\mu}+\left(\begin{array}{c}s+\alpha \\ \mu\end{array}\right) b_{\alpha-\mu}\right\} x_{\mu}+$

$$
+\left(\begin{array}{c}
s+\alpha \\
s
\end{array}\right) a_{0} x_{1+\alpha} .
$$

En comparant (4.24) avec (4.21) on trouve sans peine (4.22) avec la condition (4.23a); la démonstration du lemme pour $0 \leqq \alpha<s$ est donc achevée.

Dans ce qui suit, nous allons donc supposer $s \leqq \alpha$.

Faisant usage de l'équivalance des inégalités

$$
0 \leqq t_{1} \leqq t_{0}, \quad 0 \leqq t_{2} \leqq t_{1}, \ldots, 0 \leqq t_{k} \leqq t_{k-1}
$$

( $k \geqq 2 ; t_{0}, t_{1}, \ldots, t_{k}$ sont des nombres entiers positifs) aux inégalités $0 \leqq t_{k} \leqq t_{0}$, $t_{k} \leqq t_{k-1} \leqq t_{0}, \ldots, t_{2} \leqq t_{1} \leqq t_{0}$, on peut remplacer (3.3b) par

$$
\begin{gathered}
y_{s+\alpha}-x_{s+\alpha}=\sum_{\mu=1}^{\alpha+1}\left(\begin{array}{c}
s+\alpha \\
\mu-1
\end{array}\right) a_{\alpha+1-\mu} x_{\mu}+\sum_{\mu=0}^{\alpha}\left(\begin{array}{c}
s+\alpha \\
\mu
\end{array}\right) b_{\alpha-\mu} x_{\mu}+ \\
+\sum_{k=2}^{k^{\prime}} \sum_{t_{k}=0}^{t_{N}} \sum_{t_{k-1}=t_{k}}^{t_{0}} \ldots \sum_{t_{1}=t_{k}}^{t_{0}} \frac{1}{k !}\left(\begin{array}{c}
s+t_{k-1} \\
t_{k}
\end{array}\right)\left(\begin{array}{c}
2 s+t_{k-2} \\
s+t_{k-1}
\end{array}\right) \ldots\left(\begin{array}{c}
(k-1) s+t_{1} \\
(k-2) s+t_{2}
\end{array}\right) . \\
\cdot\left(\begin{array}{c}
k s+t_{0} \\
(k-1) s+t_{1}
\end{array}\right) a_{t_{k-1}-t_{k}} \ldots a_{t_{1-t}} a_{t_{0}-t_{1}} x_{t_{k}+k}+\sum_{k=2}^{k^{\prime}} \sum_{t_{k}=0}^{t_{0}} \sum_{t_{k-1}=t_{k}}^{t_{n}} \ldots \sum_{t_{1}=t_{k}}^{t_{n}} \frac{1}{(k-1) !} . \\
\cdot\left(\begin{array}{c}
s+t_{k-1} \\
t_{k}
\end{array}\right)\left(\begin{array}{c}
2 s+t_{k-2} \\
s+t_{k-1}
\end{array}\right) \ldots\left(\begin{array}{c}
(k-1) s+t_{1} \\
(k-2) s+t_{2}
\end{array}\right)\left(\begin{array}{c}
k s+t_{0} \\
(k-1) s+t_{1}
\end{array}\right) . \\
. a_{t_{k-1}-t_{k}} \ldots a_{t_{1}-t_{2}} b_{t_{0}-t_{1}} x_{t_{k+k-1}}, \\
\left(t_{0}=\alpha-(k-1) s\right),
\end{gathered}
$$

où $k^{\prime} \geqq 2$ est déterminé sans ambiguïté (pour $\alpha \geqq s$ fixe) par les inégalités

$$
\left(k^{\prime}-1\right) s \leqq \alpha<k^{\prime} s \text {. }
$$

a) Commençons par le cas $\mu=0$; de (4.25) on obtient immédiatement

$$
L_{s, \alpha}^{0}=\left(\begin{array}{c}
s+\alpha \\
0
\end{array}\right) b_{c}
$$

or e'est l'équation (4.22a). 
En déduisant (4.27) de (4.25) nous n'avons pas fait usage de la supposition $s>1$, or la relation (4.22a) reste également vraie pour $s=1$ [v. (4.8a)].

b) Passons au cas $\mu=1, \ldots, \alpha$. En supposant $s \leqq \alpha<2 s$, c'est-à-dire en supposant $k^{\prime}=2$, on trouve de (4.25) pour les cas

a) $s=2, \alpha=2, \beta) s=2, \alpha=3, \gamma) s>2, \alpha=s, \delta) s>2, s<\alpha<2 s$ successivement

a) $y_{4}-x_{4}=b_{2} x_{0}+\left(a_{2}+4 b_{1}+6 a_{0} b_{0}\right) x_{1}+\left(4 a_{1}+6 b_{0}+3 a_{0}^{2}\right) x_{2}+6 a_{0} x_{3}$,

в) $y_{5}-x_{5}=b_{3} x_{0}+\left(a_{3}+5 b_{2}+10 a_{0} b_{1}+10 a_{1} b_{0}\right)+\left(5 a_{2}+10 b_{1}+\right.$ $\left.+10 a_{0} a_{1}+30 a_{0} b_{0}\right) x_{2}+\left(10 a_{1}+10 b_{0}+15 a_{0}^{2}\right) x_{3}+10 a_{0} x_{4}$,

r) $y_{2 s}-x_{2 s}=b_{s} x_{0}+\left\{a_{s}+2 s b_{s-1}+\left(\begin{array}{c}2 s \\ s\end{array}\right) a_{0} b_{0}\right\} x_{1}+\left\{2 s a_{s-1}+\left(\begin{array}{c}2 s \\ 2\end{array}\right) b_{s-2}+\right.$ $\left.+\frac{1}{2}\left(\begin{array}{c}2 s \\ s\end{array}\right) a_{0}^{2}\right\} x_{2}+\sum_{\mu=3}^{s}\left\{\left(\begin{array}{c}2 s \\ \mu-1\end{array}\right) a_{s+1-\mu}+\left(\begin{array}{c}2 s \\ \mu\end{array}\right) b_{s-\mu}\right\} x_{\mu}+\left(\begin{array}{c}2 s \\ s\end{array}\right) a_{0} x_{s+1}$,

б) $y_{s+\alpha}-x_{s+\alpha}=b_{\alpha} x_{0}+\left\{a_{\alpha}+(s+\alpha) b_{\alpha-1}+\sum_{\nu=0}^{\alpha-s}\left(\begin{array}{l}s+\alpha \\ s+\nu\end{array}\right) a_{\nu} b_{\alpha-s-\nu}\right\} x_{1}+$

$$
\begin{aligned}
& +\sum_{\mu=2}^{1+\alpha-s}\left\{\left(\begin{array}{l}
s+\alpha \\
\mu-1
\end{array}\right) a_{\alpha+1-\mu}+\left(\begin{array}{c}
s+\alpha \\
\mu
\end{array}\right) b_{\alpha-\mu}+\frac{1}{2} \sum_{\nu=\mu-2}^{\alpha-s}\left(\begin{array}{l}
s+v \\
\mu-2
\end{array}\right)\left(\begin{array}{c}
s+\alpha \\
s+v
\end{array}\right) .\right. \\
& \left.. a_{\nu-\mu+2} a_{\alpha-s-\nu}+\sum_{\nu=\mu-1}^{\alpha-s}\left(\begin{array}{c}
s+v \\
\mu-1
\end{array}\right)\left(\begin{array}{c}
s+\alpha \\
s+v
\end{array}\right) a_{\nu-\mu+1} b_{\alpha-s-\nu}\right\} x_{\mu}+ \\
& +\left\{\left(\begin{array}{c}
s+\alpha \\
2 s-1
\end{array}\right) a_{s-1}+\left(\begin{array}{c}
s+\alpha \\
2 s-2
\end{array}\right) b_{s-2}+\frac{1}{2}\left(\begin{array}{c}
\alpha \\
s
\end{array}\right)\left(\begin{array}{c}
s+\alpha \\
\alpha
\end{array}\right) a_{0}^{2}\right\} x_{2-s+\alpha}+ \\
& +\sum_{\mu=3-s+\alpha}^{\alpha}\left\{\left(\begin{array}{l}
s+\alpha \\
\mu-1
\end{array}\right) a_{\alpha+1-\mu}+\left(\begin{array}{c}
s+\alpha \\
\mu
\end{array}\right) b_{\alpha-\mu}\right\} x_{\mu}+\left(\begin{array}{c}
s+\alpha \\
\alpha
\end{array}\right) a_{0} x_{\alpha+1} \cdot(4.28)
\end{aligned}
$$

De (4.28) il s'ensuit, qu'on a pour $\mu=1$; .., $\alpha$ (en supposant $s \leqq \alpha<2 s$ )

$$
L_{s, \alpha}^{\mu}=\left(\begin{array}{c}
s+\alpha \\
\mu-1
\end{array}\right) a_{\alpha+1-\mu}+\left(\begin{array}{c}
s+\alpha \\
\mu
\end{array}\right) b_{\alpha-\mu}+* L_{s, \alpha}^{\mu},
$$

où $* L_{s, \alpha}^{\mu}$ est un polynôme en $a_{0}, a_{1}, \ldots, a_{\alpha-\mu}, b_{0}, b_{1}, \ldots, b_{\alpha-1-\mu}$ satisfaisant aux conditions

a) $* L_{s, \alpha}^{2+\alpha-s}=\frac{1}{2}\left(\begin{array}{c}\alpha \\ s\end{array}\right)\left(\begin{array}{c}s+\alpha \\ \alpha\end{array}\right) a_{0}^{2}$, b) $* L_{s, \alpha}^{\mu}=0,(s \geqq 3 ; 3 \leqq \mu \leqq \alpha)$.

Supposons maintenant que dans (4.26) on ait $k^{\prime}>2$. De l'équation (4.25) qu'on peut mettre sous la forme

$$
\begin{gathered}
y_{s+\alpha}-x_{s+\alpha}=\sum_{\mu=1}^{\alpha+1}\left(\begin{array}{l}
s+\alpha \\
\mu-1
\end{array}\right) a_{\alpha+1-\mu}+\sum_{\mu=0}^{\alpha}\left(\begin{array}{c}
s+\alpha \\
\mu
\end{array}\right) b_{\alpha-\mu} x_{\mu}+ \\
+\frac{1}{2} \sum_{\mu=2}^{\alpha+2-s} \sum_{\nu=\mu-2}^{\alpha-s}\left(\begin{array}{l}
s+v \\
\mu-2
\end{array}\right)\left(\begin{array}{l}
s+\alpha \\
s+\nu
\end{array}\right) a_{\nu+2-\mu} a_{\alpha-s-\nu} x_{\mu}+\sum_{\mu=1}^{\alpha+1-s} \sum_{\nu=\mu-1}^{\alpha-s}\left(\begin{array}{l}
s+v \\
\mu-2
\end{array}\right) .
\end{gathered}
$$




$$
\begin{gathered}
\cdot\left(\begin{array}{c}
s+\alpha \\
s+v
\end{array}\right) a_{v+1-\mu} b_{\alpha-s-\nu} x_{\mu}+\sum_{k=3}^{k^{\prime}} \sum_{\mu=k}^{k+t_{0}} \sum_{t_{k-1}=\mu-k}^{t_{n}} \sum_{t_{k-2}=t_{k-1}}^{t_{0}} \ldots \sum_{t_{1}=t_{2}}^{t_{n}} \frac{1}{k !} \cdot \\
\cdot\left(\begin{array}{c}
s+t_{k-1} \\
\mu-k
\end{array}\right)\left(\begin{array}{c}
2 s+t_{k-2} \\
s+t_{k-1}
\end{array}\right) \ldots\left(\begin{array}{c}
(k-1) s+t_{1} \\
(k-2) s+t_{2}
\end{array}\right)\left(\begin{array}{c}
s+\alpha \\
(k-1) s+t_{1}
\end{array}\right) a_{t_{k-1}-\mu+k} a_{t_{k-2}-t_{k-1}} \ldots \\
\ldots a_{t_{1}-t_{2}} a_{\alpha-(k-1) s-t_{1}} x_{\mu}+\sum_{k=3}^{k^{\prime}} \sum_{\mu=k-1}^{k-1+t_{0}} \sum_{t_{k-1}}^{t_{0}} \sum_{\mu-k+1}^{t_{0}} \ldots \sum_{t_{k}-t_{k-1}}^{t_{t_{0}}} \frac{1}{(k-1) !} \cdot \\
\cdot\left(\begin{array}{c}
s+t_{t_{1}=t_{2}} \\
\mu-k+1
\end{array}\right)\left(\begin{array}{c}
2 s+t_{k-2} \\
s+t_{k-1}
\end{array}\right) \ldots\left(\begin{array}{c}
(k-1) s+t_{1} \\
(k-2) s+t_{2}
\end{array}\right)\left(\begin{array}{c}
s+\alpha \\
(k-1) s+t_{1}
\end{array}\right) \cdot \\
. a_{t_{k-1}-\mu+k-1} a_{t_{k-2}-t_{k-1}} \ldots a_{t_{1}-t_{2}} b_{\alpha-(k-1) s-t_{1}} x_{\mu} \quad\left(t_{0}=\alpha-(k-1) s\right)
\end{gathered}
$$

on déduit:

$\alpha$ Dans les produits $a_{v+2-\mu} a_{\alpha-s-v}(v=\mu-2, \ldots, \alpha-s)$ pour $\mu=2, \ldots$, $\ldots, \alpha+2-s$, il n'existe aucun facteur $a_{\alpha+1-\mu}$. S'il en existait, on devrait donc avoir ou bien $\alpha+1-\mu=v+2-\mu$, c'est-à-dire $v=\alpha-1$, ou bien $\alpha+1-\mu=\alpha-s-v$, c'est-à-dire $\nu=\mu-(s+1)$. Suivant la supposition, on a $s>1$; or le premier (deuxième) cas ne peut pas avoir lieu, parce que $v_{\max }=\alpha-s\left(v_{\min }=\mu-2\right)$.

ß) Dans les produits $a_{v+1-\mu} b_{\alpha-s-v}(v=\mu-1, \ldots, \alpha-s)$ pour $\mu=1, \ldots$, $\ldots, \alpha+1-s$, il n'existe aucun facteur $\alpha_{\alpha+1-\mu}$. S'il en existait; on devrait donc avoir $v=\alpha$. Prenant égard aux conditions $v_{\operatorname{mas}}=\alpha-s, s>1$, on voit que $v=\alpha$ n'a pas lieu. Comme $v_{\min }=\mu-1, s>1$, les produits $a_{v+1-\mu}$. . $b_{\alpha-s-\nu}$ ne contiennent même pas le facteur $b_{\alpha-\mu}$.

ү) En supposant $\mu=k, \ldots, k+\alpha-(k-1) s$. les produits

$$
a_{t_{k-1}-\mu+k} a_{t_{k-2}-t_{k-1}} \ldots a_{t_{1}-t_{2}} a_{\alpha-(k-1)_{s-t_{1}}}
$$

où

$$
\begin{gathered}
\mu-k \leqq t_{k-1} \leqq \alpha-(k-1) s, \quad \mu-k \leqq t_{k-i} \leqq t_{k-i-1} \leqq \alpha-(k-1) s \\
(i=1, \ldots, k-2)
\end{gathered}
$$

ne contiennent pas le facteur $a_{\alpha+1-\mu}$. S'il existait dans un des produits (4.32) précisément un des cas suivants devrait avoir lieu:
a) $t_{k-1}=\alpha+1-k$,
b) $t_{k-i-1}-t_{k-i}=\alpha+1-k, \quad i=1, \ldots, k-2$,
c) $t_{1}=\mu-s(k-1)-1$.

Or, d'après $(4.33)$, on devrait alors avoir $(1-s)(k-1) \geqq 0$. Mais, selon la supposition $s>1, k \geqq 3$, la dernière inégalité ne peut pas avoir lieu.

ס) En supposant $\mu=k-1, \ldots, k-1+\alpha-(k-1) s$ les produits $a_{t_{k-1}-\mu+k-1} a_{t_{k-2}-t_{k-1}} \ldots a_{t_{1}-t_{2}} b_{\alpha-(k-1) s-t_{1}}$, où $t_{1}, \ldots, t_{k-1}$ satisfont aux inégalités

$$
\begin{gathered}
\mu-k+1 \leqq t_{k-1} \leqq \alpha-(k-1) s \\
\mu-k+1 \leqq t_{k-i} \leqq t_{k-i-1} \leqq \alpha-(k-1) s, \quad(i=1, \ldots, k-2),
\end{gathered}
$$


ne contiennent ni le facteur $a_{\alpha+1-\mu}$, ni le facteur $b_{\alpha-\mu}$; la démonstration peut être donnée comme ci-dessus dans le cas $\gamma$ ).

De $\alpha)-\delta$ ) il résulte que l'équation (4.29) est vraie aussi pour $\mu=1, \ldots, \alpha$ où $\alpha$ satisfait aux conditions (4.26) avec $k^{\prime}>2$. On a donc démontré $(4.22 \mathrm{~b})$ pour chaque $\alpha>0, \mu=1, \ldots, \alpha$.

Il ne nous reste qu'à vérifier les conditions $(4.23 \mathrm{a}, \mathrm{b})$ pour $* L_{s, \alpha}^{\alpha}(\alpha \geqq s)$.

En supposant $s=2$, nous trouvons la relation $(4.23 \mathrm{~b})$ par un calcul direct de (4.30a) pour $\alpha=3,4$ et de (4.31) pour $\alpha>4$. En supposant $s>2$, nous obtenons $* L_{s, \alpha}^{\alpha}=0(\alpha \geqq s)$.

Pour $s \leqq \alpha<2 s$ l'assertion donnée plus haut est une conséquence de (4.30b). De (4.31) on voit immédiatement que pour $\alpha>2 s$ dans ${ }^{*} L_{s, \alpha}^{\alpha}$ il n'existe aucun membre quadratique en $a_{v}, b_{v}$. On peut montrer sans difficulté que, dans $* L_{s, \alpha}^{\alpha}$,il n'existe même pas de membres de degré plus haut que le deuxième. $\mathrm{Si}$, au contraire, un des produits (4.32) était contenu dans $* L_{s, \alpha}^{\alpha}$, on devrait avoir des inégalités $k \leqq \alpha \leqq k+\alpha-s-(k-2) s$, d'où on déduirait $k \leqq 1+$ $+\frac{1}{s-1}$; cela ne peut pas avoir lieu pour aucun $k$, parce que, selon la supposition, $k \geqq 3$. Pareillement, on peut démontrer que $* L_{s, \alpha}^{\alpha}$ ne contient aucun produit de la forme (4.34).

c) Enfin, il faut calculer $L_{s, \alpha}^{\alpha+1}$ pour $\alpha \geqq s$. Avant tout on voit immédiatement de (4.28) que pour $s \leqq \alpha<2 s$ la relation (4.22c) est vérifiée. Les équations (4.31) montrent que pour $\alpha \geqq 2 s$ dans $L_{s, \alpha}^{\alpha+1}$ il n'existe de produits ni de la forme $a_{\nu} a_{\lambda}$ ni de la forme $a_{\nu} b_{\lambda}$. En outre, dans $L_{s, \alpha}^{\alpha+1}$ les produits de la forme (4.32) ou (4.34) n'existent pas non plus. S'il existait, dans le coefficient $L_{s, \alpha}^{\alpha+1}$, quelque produit de la forme (4.32), il faudrait alors que les inégalités $k \leqq \alpha+$ $+1 \leqq k+\alpha-s-(k-2) s$ fussent satisfaites. Or, d'après la supposition $s>1$, on devrait avoir l'inégalité $k \leqq 1$, ce qui ne peut pas avoir lieu, parce que, selon la supposition, on a $k \geq 3$. Pareillement, on peut démontrer que dans $L_{s, \alpha}^{\alpha+1}$ il n'existe aucun produit de la forme (4.34).

Ensuite, on déduit de (4.31) que pour $\alpha>2 s(4.23 \mathrm{c})$ est vérifié également.

La démonstration du lemme est donc complète.

\section{LITTÉRATURE}

[1] E. Čech: K diferenciální geometrii prostorových křivek, Rozpravy České akademie, t. 30, 1921, No 15.

[2] E. Cech: Projektivní diferenciální geometrie, Praha, Sborník Jednoty čsl. mat. a fysiků, t. 18, 1926.

[3] E. Cech: Propriétés projectives du contact I, Spisy vyd. př́rodov. fak. Mas. university, Brno, 1928, No 91. 
[4] E. Cech: Propriétés projectives du contact II, Spisy vyd. př́rodov. fak. Mas. university, Brno, 1930, No 121.

[5] A. Urban: O styku křivek v projektivním prostoru, Časopis pro pěst. matematiky, 81, $1956,121-122$.

[6] A. Urban: Styk křivek v projektivním prostoru, Sborník První vědecké konference ČVUT, fak. strojní, 1956.

\section{Резюме}

\section{ОСНОВНАЯ ТЕОРЕМА ТЕОРИИ КАСАНИЯ КРИВЫХ}

АЛОИС УРБАН (Alois Urban), Прага.

(Поступило в редакцию 23/V 1956 г.)

При общем решении двух основных проблем теории касания кривых, т. е., во-первых, проблемы касания проекций двух кривых при проектировании из одного центра ([3]) и, во-вторых, проблемы касания проекций одной и той же кривой при проектировании из двух центров, академик Э. Чех воспользовался с выгодой теоремой, высказывающей необходимые и достаточные условия для того, чтобы две кривые, о которых предполагается, что они имеют в их общей точке, не являющейся особой точкой ни одной из этих кривых, касание порядка $s-1(s \geqq 1)$, имели в этой точке в действительности касание порядка $s+\sigma-1(\sigma \geqq 1)$; притом он поставил еще ограничивающее условие $\sigma \leqq s$ ([3]).

В работе доказана более общая теорема без указанного условия.

Отдел 2 посвящен доказательству этой теоремы для евклидова пространства (Théorème 1); найденный результат используется в отделе 3 при доказательстве основной теоремы (Théorème 2) для проективного пространства $S_{n}(n \geqq 2)$. Если $C_{1}, C_{2}$ - данные кривые пространства $S_{n}$ с параметрическими уравнениями (3.1), $A$ - их общая точка, являющаяся простой на каждой из этих кривых и соответствующая на $C_{1}\left(C_{2}\right)$ параметру $w=\stackrel{\circ}{w}(v=\stackrel{\circ}{v})$, то основная теорема теории касания кривых может быть сформулирована следующим образом (Théorème 2):

Пусть $s \geqq 1, \sigma \geqq 1-$ целье числа. Пусть $k_{0}-$ ичелое число, подобранное тал, чтобы выполнялось

$$
\left(k_{0}-1\right) s+1 \leqq \sigma \leqq k_{0} s .
$$

Пусть $C_{1}$ и $C_{2}$ имеют в точке $A$ аналитическое касание порядка $s-1$; nусть $y_{v}=x_{v}(v=0, \ldots, s-1)$.

Кривые $C_{1}$ и $C_{2}$ имеют в точке $A$ касание порядка $s+\sigma-1$ тогда и только тогда, если возмохсно подобрать такие числа $a_{v}, b_{v}(v=0, \ldots$, 
$\ldots, \sigma-1 ; a_{0} \neq-1$ при $\left.s=1\right)$, что при $k_{0}=1$ справедливо уравнение (3.2), при $k_{0}>1-$ уравнение $\left(3.3 \mathrm{a}\right.$, б), причем $t_{0}=\alpha-\left(k^{\prime}-2\right) s u k^{\prime}$ oпределено так, ито $2 \leqq k^{\prime} \leqq k_{0},\left(k^{\prime}-2\right) s \leqq \alpha<\left(k^{\prime}-1\right) s$.

В отделе 4 высказывается прежде всего теорема для случая $s=1$ (Théorème 3), которая в основном является лишь частным случаем основной теоремы (Théorème 2). При ее помощи доказывается дальнейшая теорема (Théorème 4), отличающаяся от предыдущей только тем, что вместо условия $y_{0}=x_{0}$ предполагается $y_{0}=b x_{0}(b \neq 0)$, что часто встречается при исследовании касания проекций кривых, не имеющих общей точки.

Непосредственное применение доказанных теорем к решению общих проблем теории касания кривых является, ввиду значительной сложности найденных необходимых и достаточных условий, весьма затруднительным. Но к решению некоторых частных проблем достаточно знать только расположение чисел $a_{v}, b_{v}(v=0, \ldots, \sigma-1)$ в коэффициентах при $x_{\mu}(\mu=0, \ldots$, $\ldots, s+\alpha+1 ; \alpha=0,1, \ldots, \sigma-s-1)$ в $(3,2)$ и $(3,3)$ или в $(4.4)$ и (4.5). Оказывается выгодным рассматривать случаи $s=1$ и $s>1$ в отдельности.

Для $s=1$ справедлива:

Лемма 1. Пусть уравнение (4.7) при $\alpha=0$ равносильно уравнению (4.4) и при $\alpha>0-$ уравнению (4.5); тогда имеет место (4.8), где ${ }^{*} L_{1, \alpha}^{\mu}(\mu<\alpha)-$ многочлен в $a_{v}, b_{v}, b ; * L_{1, \alpha}^{\alpha}=0$.

Аналогично для $s>1$ справедлива:

Лемма 2. Пусть (4.21) при $0 \leqq \alpha<s$ равносильно (3.2) или (3.3a), а при $s \leqq \alpha-(3.3 б) ;$ тогда имеет место (4.22), где ${ }^{\mu} L_{s, \alpha}(s \leqq \alpha, \mu<\alpha)-$ многоилен в $a_{v}, b_{v}$, для которого выполняется условие (4.23).

Найденные результаты будут использованы в дальнейших работах при решении обеих основных проблем теории касания кривых. 